\title{
Turbulent mixing driven by spherical implosions. Part 1. Flow description and mixing-layer growth
}

\author{
M. Lombardini , D. I. Pullin and D. I. Meiron \\ Graduate Aerospace Laboratories, California Institute of Technology, Pasadena, CA 91125, USA
}

(Received 12 June 2013; revised 29 January 2014; accepted 20 March 2014;

first published online 28 April 2014)

We present large-eddy simulations (LES) of turbulent mixing at a perturbed, spherical interface separating two fluids of differing densities and subsequently impacted by a spherically imploding shock wave. This paper focuses on the differences between two fundamental configurations, keeping fixed the initial shock Mach number $\approx 1.2$, the density ratio (precisely $\left|A_{0}\right| \approx 0.67$ ) and the perturbation shape (dominant spherical wavenumber $\ell_{0}=40$ and amplitude-to-initial radius of $3 \%$ ): the incident shock travels from the lighter fluid to the heavy fluid or, inversely, from the heavy to the light fluid. After describing the computational problem we present results on the radially symmetric flow, the mean flow, and the growth of the mixing layer. Turbulent statistics are developed in Part 2 (Lombardini, M., Pullin, D. I. \& Meiron, D. I. J. Fluid Mech., vol. 748, 2014, pp. 113-142). A wave-diagram analysis of the radially symmetric flow highlights that the light-heavy mixing layer is processed by consecutive reshocks, and not by reverberating rarefaction waves as is usually observed in planar geometry. Less surprisingly, reshocks process the heavy-light mixing layer as in the planar case. In both configurations, the incident imploding shock and the reshocks induce Richtmyer-Meshkov (RM) instabilities at the density layer. However, we observe differences in the mixing-layer growth because the RM instability occurrences, Rayleigh-Taylor (RT) unstable scenarios (due to the radially accelerated motion of the layer) and phase inversion events are different. A small-amplitude stability analysis along the lines of Bell (Los Alamos Scientific Laboratory Report, LA-1321, 1951) and Plesset (J. Appl. Phys., vol. 25, 1954, pp. 96-98) helps quantify the effects of the mean flow on the mixing-layer growth by decoupling the effects of RT/RM instabilities from Bell-Plesset effects associated with geometric convergence and compressibility for arbitrary convergence ratios. The analysis indicates that baroclinic instabilities are the dominant effect, considering the low convergence ratio $(\approx 2)$ and rather high $(\ell>10)$ mode numbers considered.

Key words: compressible turbulence, shock waves, turbulent mixing

\section{Introduction}

The contemporary study of accelerated density inhomogeneities began with the small-amplitude analysis of a perturbed interface by Taylor (1950). This constant acceleration environment is called the Rayleigh-Taylor (RT) instability. Taylor

† Email address for correspondence: manuel@caltech.edu 
showed that an interface separating two fluids of differing density is unstable depending on whether the acceleration is directed from the lighter to the heavier fluid. When the perturbation amplitude-to-wavelength ratio approaches one, the growth becomes nonlinear, secondary Kelvin-Helmholtz instabilities appear along the fingering structures of the distorted interface and mixing may eventually occur at small scales (e.g. Cook, Cabot \& Miller 2004).

The impulsive-acceleration analogue of the RT instability, also referred to as the Richtmyer-Meshkov (RM) instability (Richtmyer 1960; Meshkov 1969), is produced when a density interface is subjected to a shock wave. Unlike the RT instability, the occurrence of the RM instability does not depend upon the direction of impulsive acceleration; the interface is unstable whether the shock travels from the light to the heavy fluid or from the heavy to the light fluid. The RM instability is of fundamental importance in understanding the physics of inhomogeneous, compressible, turbulent flows. In particular, the transition to turbulent mixing generated by a single shock wave has been the subject of recent investigations, both experimentally (Motl et al. 2009; Orlicz et al. 2009) and numerically (Thornber et al. 2010; Lombardini, Pullin \& Meiron 2012).

While most research on both baroclinic instabilities has focused on planar geometry, little work has been done on imploding/exploding flows where density inhomogeneities are radially accelerated/decelerated, especially on the turbulent mixing generated. However, in a variety of applications, such as inertial confinement fusion (ICF) (Lindl 1998; Welser-Sherrill et al. 2008), supernova collapse (Jun, Jones \& Norma 1996; Joggerst, Almgren \& Woosley 2010), explosive detonation (Balakrishnan \& Menon 2011), underwater collapsing bubbles (Lin, Storey \& Szeri 2002) and drop impact (Krechetnikov \& Homsy 2009), these hydrodynamic instabilities take place in curved geometry. In contrast to planar geometry where only RM growth is expected to occur, converging/diverging shock-accelerated interfaces can be RT unstable as they geometrically contract or expand.

The early-time growth of these instabilities has been investigated in cylindrical (Bell 1951; Mikaelian 2005; Yu \& Livescu 2008; Lombardini \& Pullin 2009) and spherical geometries (Bell 1951; Plesset 1954; Mikaelian 1990; Kumar, Hornung \& Sturtevant 2003; Mankbadi \& Balachandar 2012). The stability analysis by Krechetnikov (2009) actually unifies some of the work previously cited by uncovering the interrelation between the RT and RM instabilities and the general effect of interfacial curvature.

Late-time mixing past the reshock event, which occurs when an initially converging shock wave impacts the interface a second time on its way out after reflecting off of the origin, has been studied for two-dimensional (2D) flows in purely azimuthal, cylindrical geometries (Zhang \& Graham 1998; Hosseini \& Takayama 2005) and (axisymmetric) spherical geometries (Glimm et al. 2002; Thomas \& Kares 2012). There are even fewer studies that consider fully three-dimensional (3D) flows where vortex stretching can now generate a wide range of turbulent scales, all the way down to Kolmogorov viscous scales. These small scales are currently unresolvable at the Reynolds numbers involved, and subgrid modelling is therefore necessary. The large-eddy simulations (LES) of Lombardini \& Deiterding (2010) considered the effect of both azimuthal and axial perturbations on the turbulent mixing driven by cylindrical implosions, while Youngs \& Williams (2008) performed monotone integrated LES (MILES) of turbulent mixing in spherical implosions. Thomas \& Kares (2012) also considered 3D mixing using a Godunov solver for the hydrodynamic part of the radiation-hydrodynamic code RAGE Gittings et al. (2008). However, their simulation was initialized from a $2 \mathrm{D}$ axisymmetric flow in a quadrant then mapped to an octant after the passage of the first reshock when the turbulent mixing is intensified. 
Youngs \& Williams (2008) calculated the mixing in a spherical sector using a spherical polar mesh. The implosion was driven by applying a varying pressure at an outer boundary which moves inward in a Lagrangian manner, the applied pressure being constant until the boundary reaches the interface at rest. The initial interface perturbation height was given by a superposition of normal modes in the polar and azimuthal directions $\theta$ and $\phi$, with a prescribed radial power spectrum, similar to what is usually done for planar interfaces. The numerical method provided the necessary artificial dissipation to capture the shocks and model the subgrid turbulent activity.

We are proposing here a different approach to simulate the turbulent mixing driven by spherical implosions. Following Lombardini \& Deiterding (2010), we perform Cartesian-grid-based LES of the two-component flow using an explicit subgrid-scale (SGS) model and a low-numerical dissipation advection scheme activated in the turbulent regions $(\S 2)$. As explained in $\$ 3$, the computational domain considered is either an octant of a sphere or a full sphere; the initial converging shock is set up as a self-similar, radially symmetric solution of the Euler equation. An analysis using spherical harmonics, which is detailed in Part 2 (Lombardini, Pullin \& Meiron 2014), is used to construct the pre-shock interfacial perturbations. We investigate two canonical configurations: the light fluid enclosed by the spherical interface, or vice versa. Various results are exposed in subsequent sections, focusing on the fundamental differences between the two configurations, and highlighting the unique aspects of the spherical geometry compared with the planar one. In $\S 4$ we analyse the main wave interactions through a study of the unperturbed, radially symmetric flow. The 3D perturbed flow is illustrated in $\S 5$, with an emphasis on the mean flow. The growth of the mixing layer is finally interpreted in $\$ 6$ using conclusions from the radially symmetric flow and from a linear stability analysis.

\section{Computational approach}

To be able to computationally represent the large dynamical range of scales produced in such compressible, high-Reynolds-number environments, we consider, as described in Lombardini et al. (2011), the two-component Favre-filtered Navier-Stokes equations governing the transport of the filtered density $\rho$, momentum $\rho u_{i}$ and total energy $E$ of the mixture, and the filtered heavy-fluid partial density $\rho \psi$, with $\psi$ the heavy-fluid mass fraction. We explicitly model the subgrid terms representing unresolvable flow features, i.e. below a fixed cutoff scale taken here as the finest grid size $\Delta$ in the computational domain. The SGS representation is essentially an extension of the stretched-vortex model of Misra \& Pullin (1997) to compressible, multicomponent flows, in which the small-scale mixing is described by the stirring of a passive scalar transported under the flow of the axisymmetric vortex Pullin (2000).

Shock waves are captured numerically using a weighted essentially non-oscillatory (WENO) scheme and, in that sense, the shock thickness is the only physical scale in the problem that is neither resolved computationally nor modelled physically. Away from shocks, the numerically diffusive WENO scheme reverts dynamically to a low-numerical dissipation, tuned centre-difference (TCD) scheme optimal for the accurate computation of regions of turbulent mixing where the SGS model activates itself Hill \& Pullin (2004). The switching of advection schemes is performed using a robust shock sensor Lombardini (2008). To reduce nonlinear instabilities, e.g. of the aliasing type, we avoid any filtering or numerical damping techniques which could alter the computation of high-Reynolds-number turbulence, and prefer to rewrite the TCD discretization of the convective terms in the momentum, energy 
and scalar equations in an energy-conserving (skew-symmetric) form Honein \& Moin (2004). Temporal stability is achieved by the use of the optimal third-order strong-stability-preserving Runge-Kutta time-stepping scheme of Gottlieb, Shu \& Tadmor (2001).

In the spherically converging RM flow presently studied, it is computationally advantageous to dynamically refine shock waves and geometrically contracting/ expanding turbulent regions. This is undertaken using the block-structured, adaptive mesh refinement (AMR) algorithm of Berger \& Colella (1989) as implemented by Deiterding (2005). The above numerical method is formulated on uniform Cartesian grids and is effectively applied to each subgrid of the AMR hierarchy. Thanks to a refinement criterion based on the local density and mass fraction gradients, we enforce that the evolution of the various shocks and the entire mixing zone are solved discretely on adaptive grids refined to the maximum level (i.e. with grid cell of size $\Delta$ ), at all times in the simulation.

Owing to the radial symmetry of the problem, we investigate various statistics on spherical surfaces of radius $r$. Cartesian AMR data are first interpolated over a regular Gaussian grid, i.e. at the points of spherical coordinates

$$
\left(r, \theta_{i}, \phi_{j}\right)=\left(r, i \frac{\pi}{N_{\theta}}, j \frac{2 \pi}{N_{\phi}}\right), \quad i=0, \ldots, N_{\theta}(r), j=0, \ldots, N_{\phi}(r)-1,
$$

where $N_{\theta}(r)=N_{\phi}(r) / 2=\lceil\pi r / \Delta\rceil$. This ensures that the finest resolved scales are retained during the interpolation process. Surface-interpolated quantities $Q\left(r, \theta_{i}, \phi_{j} ; t\right)$ will be used for spectral statistics in $\$ 4$ of Part 2, or for the discrete evaluation of spherical-surface averages formally defined as

$$
\langle Q\rangle(r, t)=\frac{1}{4 \pi} \oiint_{\Omega} Q(r, \theta, \phi ; t) \mathrm{d} \Omega .
$$

where $\mathrm{d} \Omega=\sin \theta \mathrm{d} \theta \mathrm{d} \phi$ is the local infinitesimal solid angle. Fluctuations from $\langle Q\rangle$ and from Favre-like surface averages $\widetilde{Q}=\langle\rho Q\rangle /\langle\rho\rangle$, where $\rho$ is the density of the mixture, are given by

$$
Q^{\prime}(r, \theta, \phi ; t)=Q(r, \theta, \phi ; t)-\langle Q\rangle(r, t), \quad Q^{\prime \prime}(r, \theta, \phi ; t)=Q(r, \theta, \phi ; t)-\tilde{Q}(r, t) .
$$

\section{Problem set-up}

\subsection{Geometry}

We consider two computational domains: an entire spherical volume $\mathscr{V}$, for the production of spectral statistics taken within the mixing-layer centre, as well as an octant of a sphere $\mathscr{V}_{1 / 8}$, which is less computationally expensive, for the extensive processing of various surface averages (simply replace $4 \pi$ by $\pi / 2$ in $(2.2)$ ):

$$
\begin{aligned}
\mathscr{V} & =\left\{(r, \theta, \phi) \mid r_{i n t} \leqslant r \leqslant r_{e x t}, \quad 0 \leqslant \theta \leqslant \pi, 0 \leqslant \phi<2 \pi\right\}, \\
\mathscr{V}_{1 / 8} & =\left\{(r, \theta, \phi) \mid r_{i n t} \leqslant r \leqslant r_{e x t}, \quad 0 \leqslant \theta \leqslant \pi / 2,0 \leqslant \phi \leqslant \pi / 2\right\} .
\end{aligned}
$$

A 2D view of $\mathscr{V}_{1 / 8}$ is depicted in figure 1. In both domains, an interior spherical reflective wall of radius $r_{i n t}$ is used to regularize the centre, while inflow/outflow 


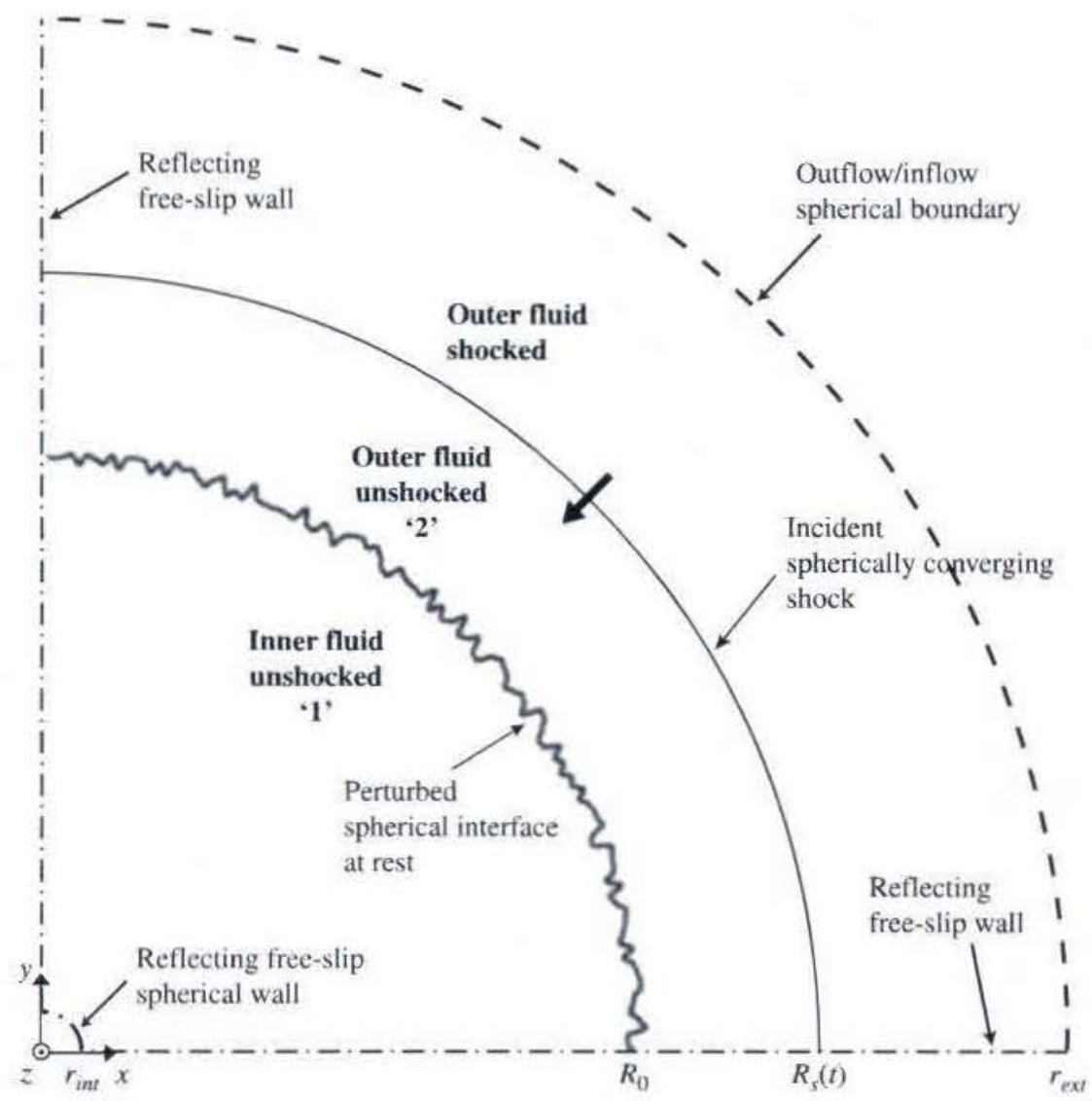

FIGURE 1. Planar cut $z=0$ of the simulation in an octant: an incident converging shock of Mach number $M_{S}(t)$ and position $r=R_{S}(t), t \leqslant t_{0}$, impacts at $t=t_{0}$ a perturbed, spherical interface at mean position $r=R_{0}$ separating two quiescent fluids of different, uniform densities $\rho_{1_{0}}$ and $\rho_{2_{0}}$.

boundary conditions are prescribed on an exterior spherical boundary of radius $r_{\text {ext }}$. A ghost fluid approach (Fedkiw et al. 1999) is utilized to numerically incorporate the non-Cartesian boundary conditions arising at the interior and exterior boundaries. For practical reasons, we choose $r_{i t}=R_{0} / 40$ and $r_{e x t}=(\pi / 2) R_{0}$, where $R_{0}$ is the initial position of the unperturbed interface. The region around $r_{i m t}$ is refined to the maximum level at all times. The base grid resolution is $128^{3}$ for $\mathscr{V}$ and $64^{3}$ for $\mathscr{V}_{1 / 8}$, on which two additional levels of refinement can be applied, each with a refinement factor of two in each Cartesian direction. As a result, the finest resolution (if the entire domain were resolved by the highest level of refinement in each direction) is $512^{3}$ for $\mathscr{V}$ and $256^{3}$ for $\mathscr{V}_{1 / 8}$, the finest grid size being then $\Delta \simeq 0.006 R_{0}$ for both domains. For $\mathscr{V}_{1 / 8}$, it is assumed that the shock-wave/boundary-layer interaction does not play a dominant role in the growth of the turbulent mixing zone and free-slip boundary conditions can be applied at the reflective walls $x=0, y=0$ and $z=0$.

\subsection{Converging shock}

The flow behind the spherically converging shock is initialized with an approximate solution of the radially symmetric Euler equations Chisnell (1998), which gives $\left(\rho, u_{r}, p\right)$, normalized by the density, radial velocity and pressure immediately behind 


$$
\text { (a) } \begin{aligned}
\mathrm{Air} & \rightarrow \mathrm{SF}_{6} \\
A_{0} & \approx 0.667 \\
M_{S_{0}} & =1.2
\end{aligned}
$$
(b) $\mathrm{SF}_{6} \rightarrow$ air
$A \approx-0.667$
$M_{S_{0}} \approx 1.222$

TABLE 1. Table of runs for the light-heavy and heavy-light configurations considered indicating pre-shock Atwood ratios and incident shock Mach number at impact $t=t_{0}$.

the shock, respectively, as a function of the similarity variable $r / R_{S}(t)$, where the shock position $R_{S}(t)$ is characterized by a similarity exponent $n(\gamma)$ Guderley (1942). For a shock travelling in air, $\gamma \approx 1.40$ gives $n \approx 0.717$, while in $\mathrm{SF}_{6}, \gamma \approx 1.09$ and $n \approx 0.799$. The self-similar structure has been preliminary confirmed by simulations of a single converging shock. In particular, $n$ has been computed before and after reflection at the centre Lombardini \& Pullin (2009). Initializing the shock using Chisnell's self-similar solution not only avoids spurious waves that would appear if setting up the shock as a Riemann problem solution for the strictly axisymmetric shock-implosion process, but also leaves the shock thickness as the only intrinsic length scale. Chisnell's solution is also used as a time-dependent inflow boundary conditions at $r=r_{\text {int }}$, until the exploding reshock exits the computational domain, after which zero-gradient boundary conditions are applied.

We define an initial Atwood ratio $A_{0}=\left(\rho_{1_{0}}-\rho_{2_{0}}\right) /\left(\rho_{1_{0}}+\rho_{2_{0}}\right)$, where $\rho_{1_{0}}$ and $\rho_{2_{0}}$ denote the initially uniform densities of the pure fluid (i.e. as the mass fraction $\psi=0$ or 1) respectively contained within the sphere of radius $R_{0}$ and exterior to this sphere. For an air- $\mathrm{SF}_{6}$ gas combination with equal initial temperatures, $A_{0}$ is simply given by the ratio of molecular weights: $\left|A_{0}\right| \approx 0.667$. The converging shock is said to impact a density interface in a light-heavy fashion when $A_{0}>0$ and in a heavy-light fashion when $A_{0}<0$. In the light-heavy configuration, the shock is positioned behind the slightly perturbed interface such that its Mach number at impact is $M_{S_{0}}=1.2$. The initial perturbation shape being fixed, the impact Mach number in the heavy-light shock interaction is chosen such that the perturbation grows with the same small-amplitude growth rate as in the light-heavy refraction, i.e. matching $k\left(a_{0} A_{0}+a_{0}^{+} A_{0}^{+}\right) \Delta u$, where $\Delta u$ is the change in radial velocity due to shock refraction, $a_{0}$ is the pre-shock perturbation amplitude and ' + ' denotes post-shock quantities. Vandenboomgaerde, Mügler \& Gauthier (1998) have shown that this adjustment of Richtmyer's original formula applies well to both light-heavy and heavy-light configurations. This gives a Mach number $M_{S_{0}} \approx 1.222$ at the heavy-light impact. Light-heavy and heavy-light configurations are summarized in table 1.

\subsection{Initial interfacial perturbation}

We describe here how to build an initially isotropic perturbation at a spherical density interface of radius $R_{0}$ (i.e. no preferred directions when moving along the spherical surface), with the idea that the turbulent mixing induced by the passage of concentric pressure waves would remain somehow isotropic, and could then be analysed assuming statistical isotropy on a sphere.

We define by $r-\zeta_{0}(\theta, \phi)=0$ the perturbed surface where light (air) and heavy $\left(\mathrm{SF}_{6}\right)$ fluids have equal mass fractions. The heavy-fluid mass fraction field $\psi(r, \theta, \phi ; t)$ initially takes the form of a hyperbolic tangent profile centred at $\zeta_{0}(\theta, \phi)$ and with characteristic thickness $L_{\psi_{0}}=0.002 R_{0}$. This is large enough to ensure the resolution 
of the diffuse interface given by

$$
\begin{aligned}
\psi\left(r, \theta, \phi ; t_{0}\right) & =\frac{1}{2}\left\{1-\frac{\left|A_{0}\right|}{A_{0}} \tanh \left[\frac{r-\zeta_{0}(\theta, \phi)}{L_{\psi_{0}}}\right]\right\} . \\
\zeta_{0}(\theta, \phi) & =R_{0}-a_{0}\left|f\left(R_{0}, \theta, \phi\right)\right|,
\end{aligned}
$$

where $f\left(R_{0}, \theta, \phi\right)$ is a perturbation field. Our experience with RM simulations has motivated the use of the absolute value of $f$ in $(3.2 b)$ : the singularity at the zeros of $|f|$ corresponds to a spread in the wavenumber space that encourages earlier nonlinear growth. In our early planar RM investigations, we had also found that the absolute value models well the regularity of the membrane used in the shocktube experiments of Vetter \& Sturtevant (1995). In order to build a homogeneous, isotropic perturbation field, $f$ is decomposed using the spherical harmonics basis as

$$
f\left(R_{0}, \theta, \phi\right)=\sum_{\ell=0}^{\infty} \sum_{m=-\ell}^{\ell} f_{\ell m} Y_{\ell m}(\theta, \phi),
$$

with coefficients

$$
f_{\ell m}=\sqrt{(2 \ell+1) C_{\ell}} \frac{\cos \left(2 \pi \omega_{\ell}^{m}\right)}{\sqrt{\sum_{j=-\ell}^{\ell} \cos \left(2 \pi \omega_{\ell}^{j}\right)^{2}}},
$$

where $\omega_{i}^{j}$ are randomly generated numbers in [0.1], and $C_{\ell}$ are given by

$$
C_{\ell}=\frac{1}{4\left(2 \ell_{0}+1\right)} \frac{1}{\sigma_{0} \sqrt{2 \pi}} \exp \left[-\frac{\left(\ell-\ell_{0}\right)^{2}}{2 \sigma_{0}^{2}}\right] .
$$

The perturbation is therefore represented by a wavepacket of modes with random phase and so-called 'angular power spectrum' $C_{\ell}$. We refer to $\S 4$ of Part 2 for the mathematical derivation of $f$. In (3.5), we have chosen a narrow Gaussian profile with dominant spherical wavenumber $\ell_{0}=40$ and variance $\sigma_{0}=\ell_{0} / 30$. For an entire sphere of radius $R_{0}$, the choice for $\ell_{0}$ would roughly correspond to 40 wavelengths in both orthogonal directions of a plane of extent $2 \sqrt{2} \pi R_{0} \times 2 \sqrt{2} \pi R_{0}$ (10 wavelengths for the planar analogue of an octant), i.e. an equivalent radial wavenumber $\kappa_{0}=\ell_{0} / R_{0}$; $\sigma_{0}$ is such that the width of the Gaussian, $\ell_{0}-3 \sigma_{0} \lesssim \ell \lesssim \ell_{0}+3 \sigma_{0}$, is well within the space of resolved spherical wavenumbers. In (3.5), the normalization constant in front guarantees that the variance of $a_{0} f$ matches the interface displacement variance of a single-mode, planar corrugation of maximum amplitude $a_{0}$ and wavenumber $k_{0}=\ell_{0} /\left(\sqrt{2} R_{0}\right)$ in both orthogonal directions of that plane. We fix $a_{0}=0.03 R_{0}$, i.e. an initial amplitude-to-wavelength ratio of approximately $20 \%$, large enough to precipitate the nonlinear development of the perturbation. The initial perturbation shape obtained is depicted in figure 2 .

Instead of employing a Gaussian power spectrum, which decays exponentially at high wavenumbers, numerical studies of planar RM/RT flows have often considered a broadband combination of modes satisfying a power-law decaying spectrum $\propto \kappa^{-q}$, $q>0$ (e.g. Thornber et al. 2010). This perturbation shape is of practical importance as it is representative of the measured surface finish of an ICF capsule Barnes et al. (2002). For simplicity, we presently focus on a narrowband Gaussian spectrum and 


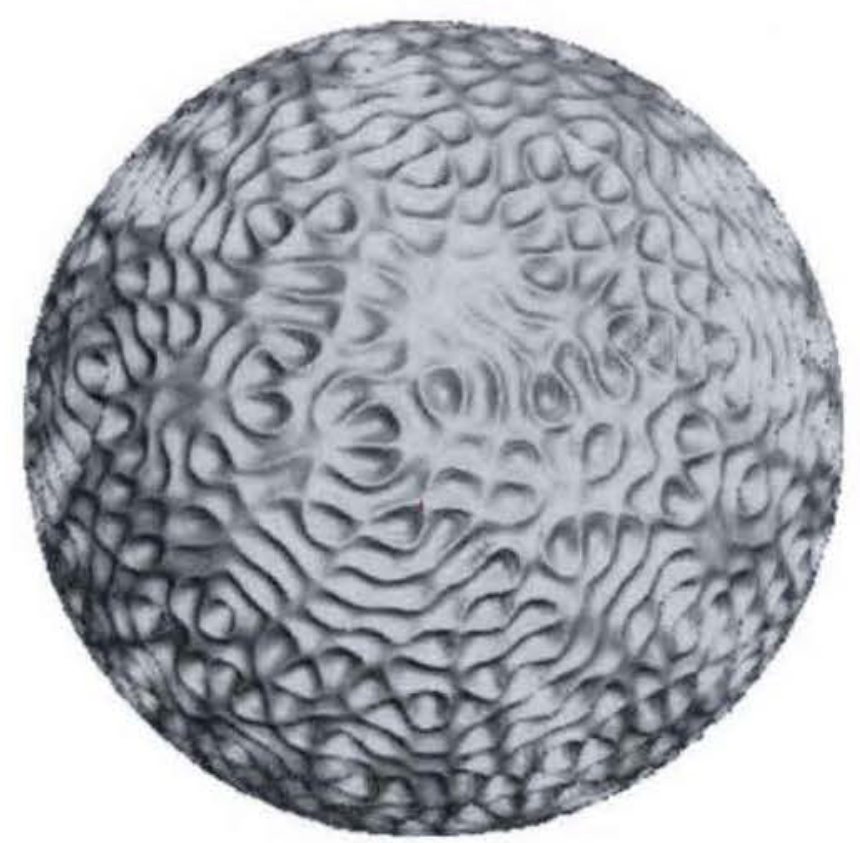

FIGURE 2. (Colour online) Initial isosurface of equal mass fractions. The statistically isotropic initial perturbation field has a characteristic amplitude $a_{0}=0.03 R_{0}$ and a shape given by a Gaussian angular power spectrum with dominant spherical wavenumber $\ell_{0}=40$ and variance $\sigma_{0}=\ell_{0} / 30$.

leave for future work the study of the influence of multimode initial perturbations on the growth of a spherical mixing layer.

Using a decomposition in terms of a spherical harmonics basis allows one to: (i) avoid the pole singularity $\theta=0$; (ii) keep a homogeneous characteristic wavenumber $\kappa_{0}$ along the $(\theta, \phi)$-manifold; (iii) inject wavenumbers around $\kappa_{0}$ that can slightly alter the symmetry as well as stimulate nonlinear mode coupling and hence the mixing. Another way to design a perturbation that satisfies (i) and (ii), but that is not strictly speaking statistically isotropic, would be to consider for example $f \propto \sin \left(k_{0} \theta\right) \sin \left[k_{0} \sin (\theta) \phi\right]$, i.e. a single-mode perturbation with constant wavenumber $k_{0}$ in the directions $\theta$ and $\phi$. However, this perturbation function would not work well for the whole sphere because it is not periodic in the azimuthal direction.

\subsection{Summary of initial conditions}

The vector of conserved quantities $(\rho, \rho \boldsymbol{u}, E, \rho \psi)$ for a mixture of two ideal gases is initialized in the simulation just before impact (i.e. $t=t_{0^{-}}$), using the following choice of $(\rho, \boldsymbol{u}, p, \psi)$. The couple $(\boldsymbol{u}, p)$ is equal to $\left(\mathbf{0}, p_{0}\right)$ ahead of the shock $r<R_{S}\left(t_{0^{-}}\right)$, and given by the pressure and radial velocity of Chisnell (1998) for $r \geqslant R_{S}\left(t_{0^{-}}\right)$. The field $\psi$ is given by $(3,2 b)$ and, ahead of the shock $r<R_{S}\left(t_{0^{-}}\right), \rho$ is then directly derived from $\psi$ assuming continuity of pressure and temperature across the interface. In particular, at $\rho=\min \left(\rho_{1_{0}}, \rho_{2_{0}}\right)$ as $\psi=0$ (pure light fluid), and $\rho=\max \left(\rho_{1_{0}}, \rho_{2_{0}}\right)$ as $\psi=1$ (pure heavy fluid). For $r \geqslant R_{S}\left(t_{0^{-}}\right), \rho$ is specified following Chisnell (1998).

\section{The radially symmetric flow}

In this section, we inspect the radially symmetric flow, i.e. when the interface is unperturbed. An $(r, t)$ wave diagram is obtained from one-dimensional simulations 
(using appropriate geometric source terms) for both light-heavy and heavy-light initial configurations. This wave diagram, depicted in figure 3 , is useful for the prediction of RM- and RT-unstable scenarios at the perturbed interface. Depending on whether the configuration is of light-heavy or heavy-light type, various waves can be produced and a labelling scheme is needed. The first uppercase letter can either be ' $I$ ', which stands for 'incident', ' $R$ ' for 'reflected' or ' $T$ ' for 'transmitted'. The second uppercase letter indicates the nature of the wave: ' $S$ ' for 'shock' or ' $R$ ' for 'rarefaction'. The number following refers to the time-wise order of appearance. For example, 'RS0' labels the reflected shock from the initial shock interaction at $t=t_{0}$, 'TS1' the transmitted shock from the first reshock at $t_{\text {Resl }}$ (i.e. the second shock interaction), etc. Here 'Resi' represents the reshock wave during the $i$ th reshock interaction and ' $C$ ' denotes the unperturbed density interface, or contact wave, with position $r=R(t)$ for $t \gtrsim t_{0}$; in particular, $R\left(t_{0}\right) \equiv R_{0}$. Since we allow the interface to be diffuse, $R(t)$ is formally defined by $\psi(R, t)=0.5$.

In the light-heavy case (figure $3 a$ ), IS 0 slows down during the initial light-to-heavy wave refraction, the light fluid must contract to maintain mechanical equilibrium at the interface, and consequently a shock RS0 is reflected. The shocked interface is accelerated inward, following behind TS0 that converges towards the origin and reflects off of it. The resulting outgoing shock Res1 reshocks the interface a first time at $t_{\text {Resi }}$. In this heavy-to-light refraction, the main shock accelerates (TS1), and the heavy fluid must expand to satisfy mechanical equilibrium at the interface: a spherical rarefaction wave, RR1, is therefore reflected back towards the origin. In addition, a secondary, inward-facing shock 'sRS1' forms at the tail of the rarefaction wave (figure 4). This only appears in spherical and cylindrical flows, and does not arise in planar geometries such as flows in a constant cross-section shocktube with an endwall. The reshock event in planar flows is solely characterized by a reflected rarefaction wave and a transmitted shock separated by a region of uniform velocity and pressure. The rarefaction wave then reverberates back and forth between the wall and the contact surface (e.g. Lombardini et al. 2011). In curved geometries, however, the fluid processed by the spherical rarefaction wave must expand to lower pressures than those reached through an equivalent planar rarefaction, because of the geometric expansion. To accommodate this 'over-expansion', a compression wave is thus necessary to connect the pressure behind the transmitted shock with the pressure at the rarefaction tail. This is mathematically confirmed by the crossing of the characteristic curves of slope $\mathrm{d} r / \mathrm{d} t=u_{r}-a$ ( $u_{r}$ is the particle velocity and $a$ the sound speed). A similar shock formation arises in the flow past the detonation of a spherical charge (Wecken 1950), when a shock travelling down a uniform tube meets an area change (Friedman 1960), or in the radial dam-break problem in the context of the shallow-water equations. The shock sRS1 grows from zero strength as it moves inward through the heavy fluid, although it is initially swept outward in space. It does not acquire a net inward velocity until the rarefaction wave is nearly exhausted, when the rarefaction head has reached the centre. The shock then implodes on the origin and is reflected outward to the contact surface, defining a second reshock event at $t_{\text {Res } 2}$. Note that the interface is swept outward following the first reshock, but slowly decelerates until it starts moving inwards due to mass conservation (the fluid inside being compressed by the secondary shock). The heavy fluid being still denser than the fluid immediately outside, a similar heavy-to-light refraction occurs at $t_{\text {Res } 2}$ and a new reflected shock sRS2 is formed. Progressively weaker reshocks 'Resi' follow from the refractions at the contact and reflections at the origin.

In the heavy-light configuration (figure $3 b$ ), IS0 first interacts with the spherical interface in a heavy-to-light fashion, generating a transmitted shock TS0 and reflected 
(a)

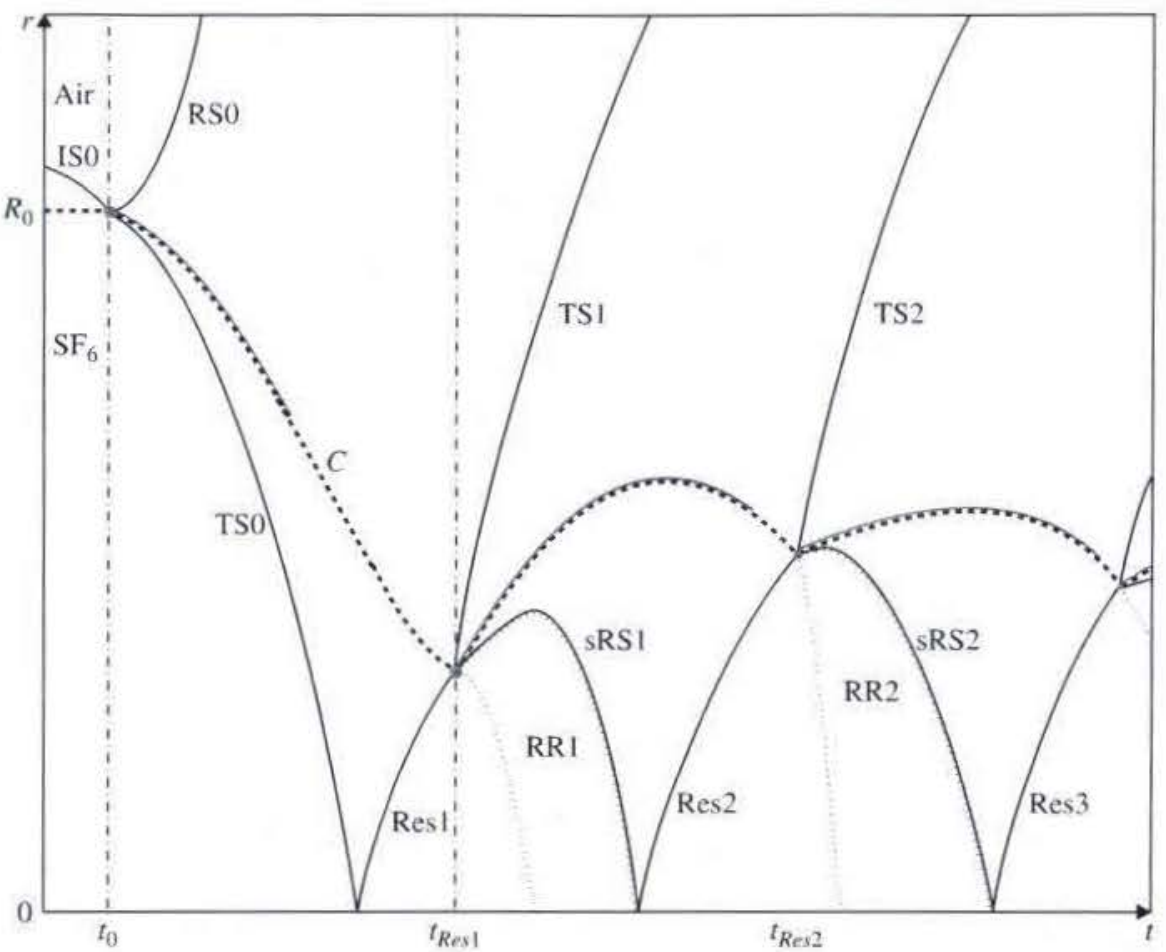

(b)

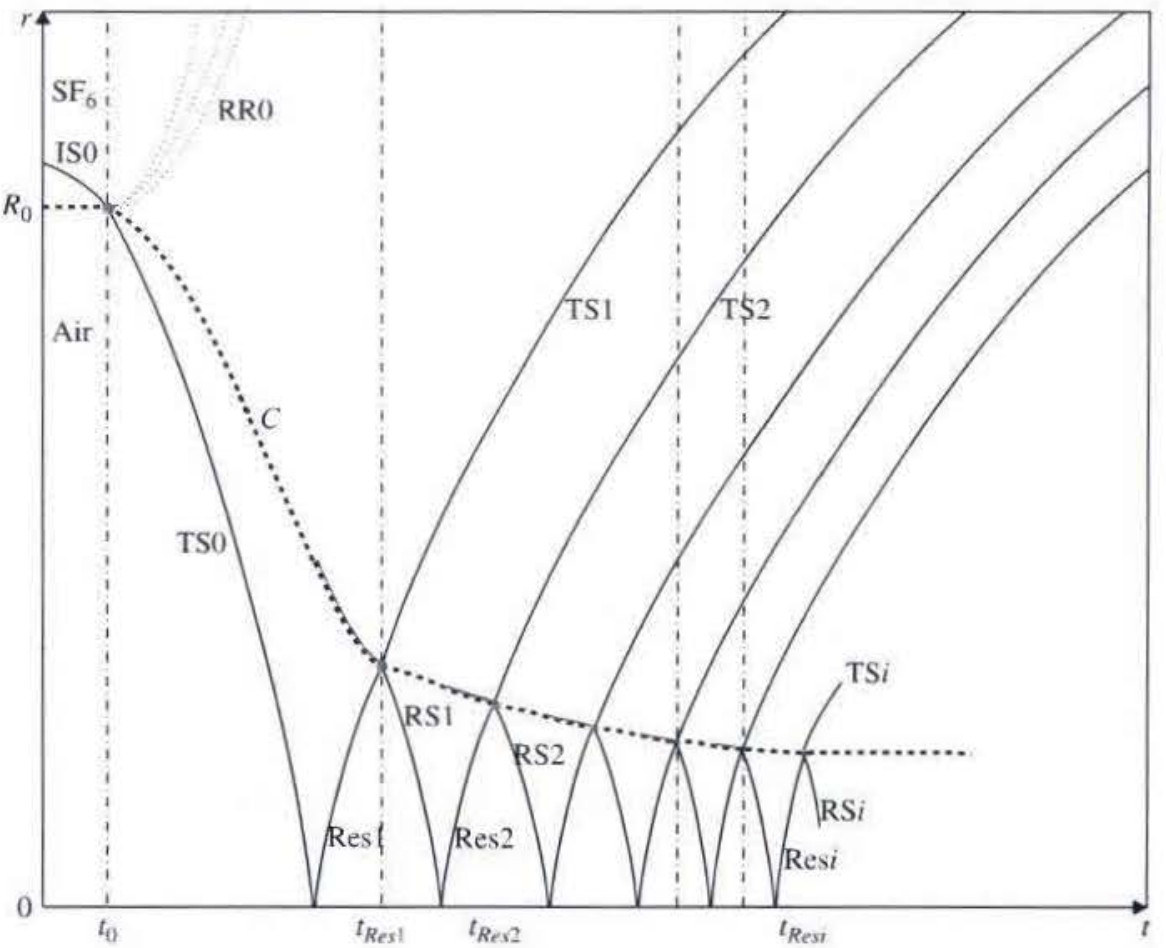

FIGURE 3. Sketch of the $(r, t)$ wave diagram for the interaction of an incident converging shock IS0 with a $(a)$ light-heavy air $\rightarrow \mathrm{SF}_{6}$ and $(b)$ heavy-light $\mathrm{SF}_{6} \rightarrow$ air density interface or contact wave $\mathrm{C}$, at $t=t_{0}$. The position of the unperturbed interface $r=R(t)$ is drawn as a thick dashed curve, the various shocks as solid curves, and the head and tail of the rarefaction waves as dotted curves. The red dots represent occurrences of potential RM instability, and the red curves represent RT-unstable regions. 


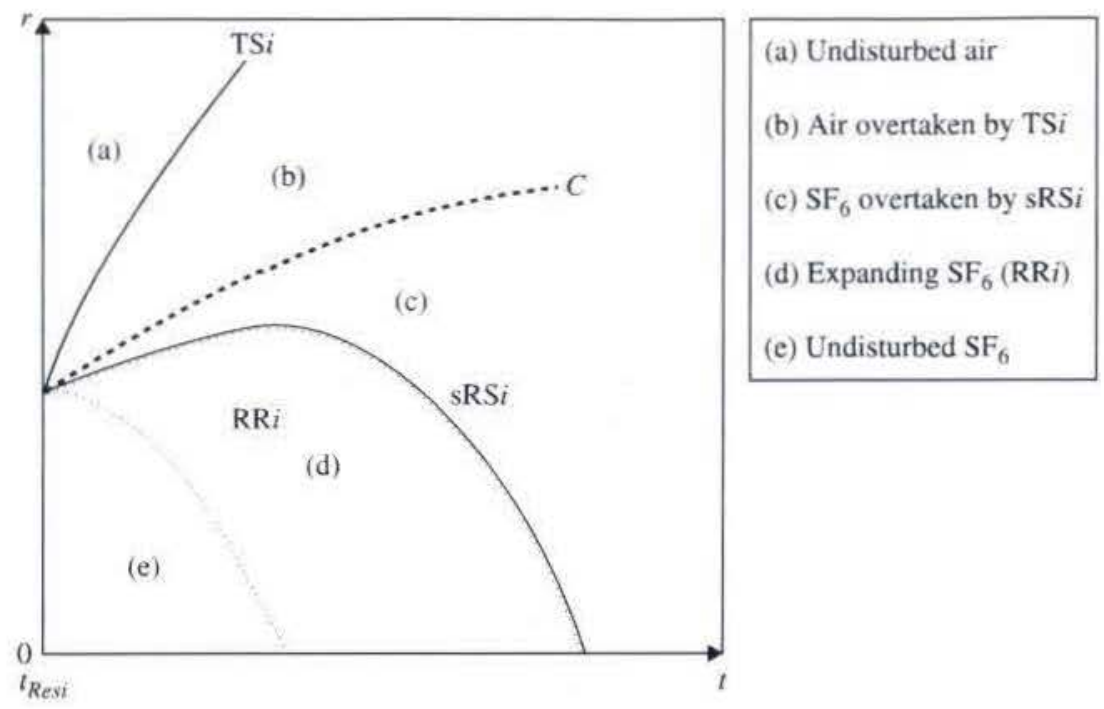

FIGURE 4. Exploding phase following a heavy-to-light reshock interaction observed in the light-heavy air $\rightarrow \mathrm{SF}_{6}$ configuration (close-up of figure $3 a$ for $t \geqslant t_{\text {Resi }}$ ).

rarefaction RR0. The interface is reshocked in a light-to-heavy fashion at $t_{\text {Res } 1}$ : the main shock, TS1, is slowed down; the converging interface decelerates as well; a reflected shock, RS1, is produced, similarly to what would be observed in the equivalent planar geometry. This shock reflects off of the origin and reshocks the interface a second time. Repeated reshocks of decreasing intensity follow until the interface reaches a constant radial position.

Light-heavy and heavy-light configurations are similar in the way the interface is reshocked multiple times. In contrast, the planar light-heavy configuration is characterized by the interaction of a rarefaction wave with the contact surface, following the first reshock. The rarefaction wave continuously deposits energy into the mixing layer over the course of its passage from head to tail, as opposed to the impulsive deposition of energy due to the primary reshock and the successive reshocks observed in the spherical geometry. In addition to RM instabilities induced by each shock interaction, RT-unstable regions exist as a result of the interface acceleration/deceleration phases peculiar to the radial geometry: when the heavy fluid is placed inside, the interface is RT unstable if it accelerates inward or decelerates outward (i.e. $\ddot{R}<0$ ); when the light fluid is inside, RT-unstable regions correspond to the interface decelerating inward or accelerating outward (i.e. $\ddot{R}>0$ ). These regions are highlighted in red in figure 3. RT effects are even more important in that the magnitude of the acceleration is large. Because of the 'oscillating' behaviour of its radial trajectory, the light-heavy interface is more predisposed to RT instabilities than the heavy-light interface whose trajectory looks more 'monotonic' in time.

We finally note that, for comparable initial growth rates, the resulting heavy-light reshock interactions occur earlier than their light-heavy equivalents, the shocks travelling faster through the light fluid than the heavy fluid.

\section{The 3D flow}

The initial density interface $C$ is now perturbed and we investigate the 3D mixing resulting from an imploding impact. Figure 5 shows the evolution of 
(a)

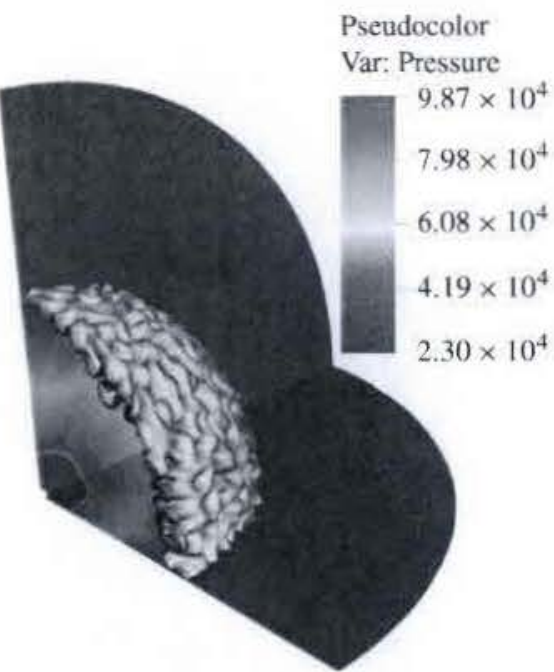

(b)

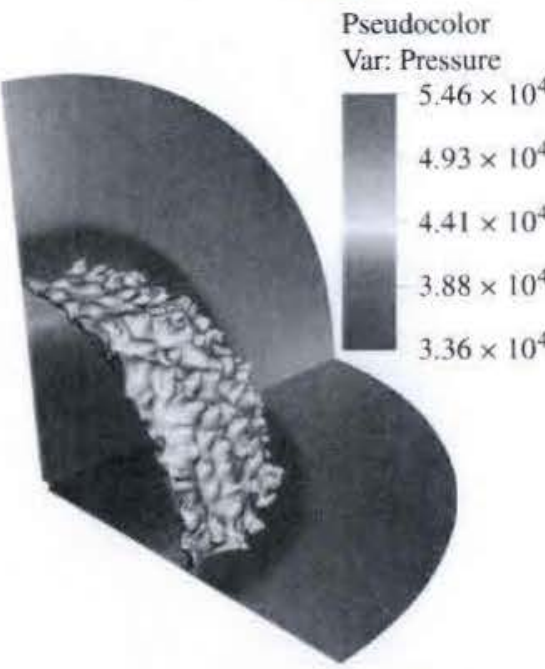

(c)

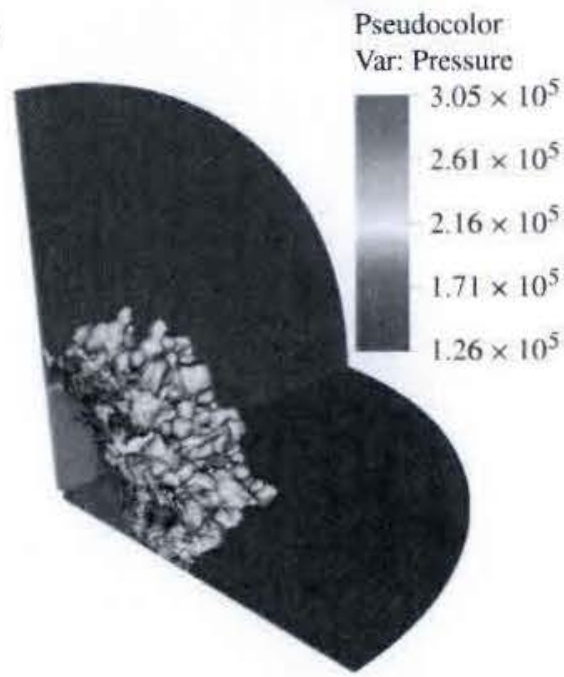

$\left(a^{\prime}\right)$

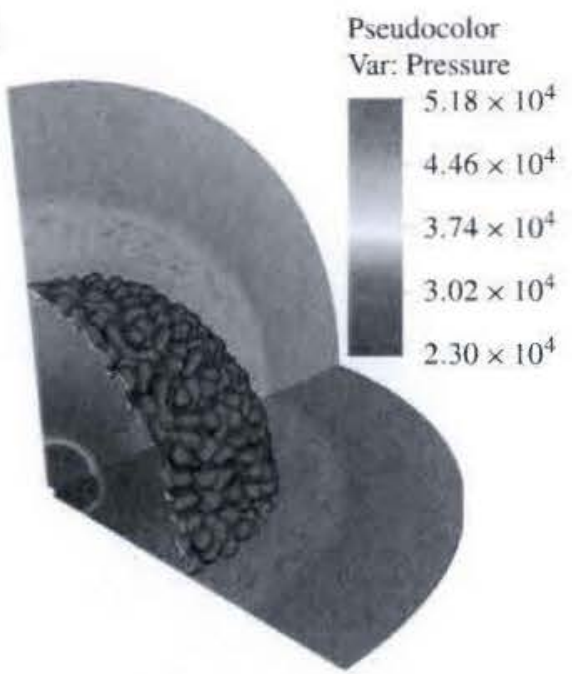

(b')

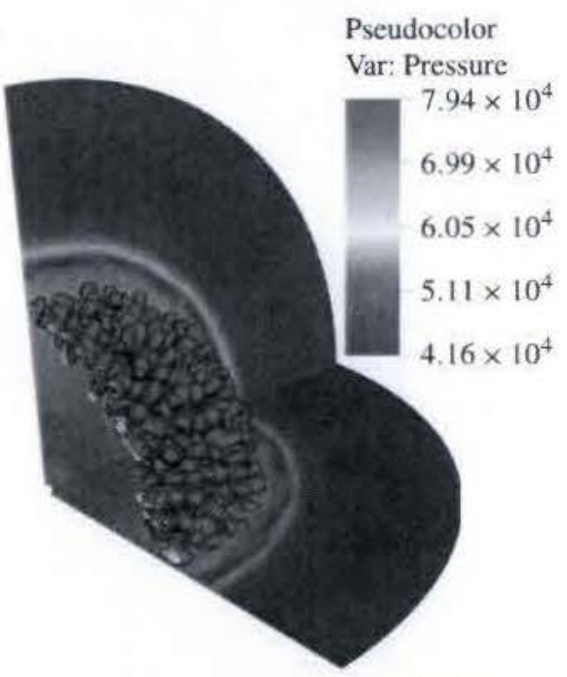

$\left(c^{\prime}\right)$

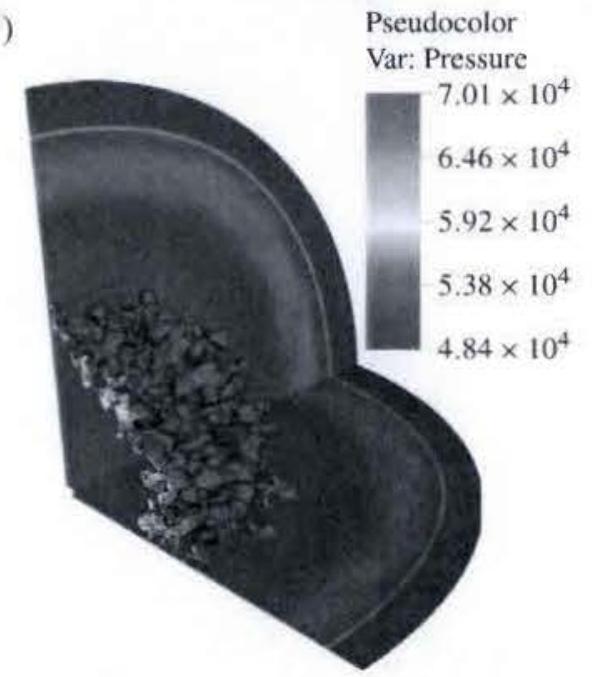

FIGURE 5. Isosurfaces of the heavy-fluid mass fraction (cyan blue for $25 \%$, green for $50 \%$ and red for $75 \%)$ and contours of pressure, at three different times: $(a)$ or $\left(a^{\prime}\right)$ $t \approx t_{\text {Res } 1} / 2 ;(b)$ or $\left(b^{\prime}\right) 1.5 t_{\text {Res } 1} ;(c)$ or $\left(c^{\prime}\right) 3 t_{\text {Resl }}$. Cases air $\rightarrow \mathrm{SF}_{6}(a, b, c)$ and $\mathrm{SF}_{6} \rightarrow$ air $\left(a^{\prime}, b^{\prime}, c^{\prime}\right)$. 
isosurfaces of the mass fraction and pressure contours on two orthogonal planes intersecting the origin. In the imploding phase (figures $5 a$ and $\bar{a}^{\prime}$ ), the interface travels inwards, following the transmitted shock TSO. The reflected shock RS0 produced by the interaction of the $M_{S}=1.2$ incident shock with the lightheavy interface has a weak Mach number $\approx 1.06$ and is therefore not noticeable in figure $5(a)$ considering the pressure range used for visualization. However, the reflected rarefaction wave RR0 from the initial heavy-to-light interaction is visible in figure $5\left(a^{\prime}\right)$. On the same subfigure, we also notice that a phase reversal, characteristic of heavy-to-light interactions, has occurred: the initial spikes (which refer to fingering structures of heavy fluid penetrating light fluid) become bubbles (i.e. pockets of light fluid penetrating heavy fluid), and initial bubbles turn into spikes. The post-reshock state of the flow is depicted in figures $5(b)$ and $5\left(b^{\prime}\right)$. In the light-heavy configuration (figure $5 b$ ), the heavy-to-light reshock has caused a phase reversal while a reflected shock SRS1 is forming, as predicted in the previous section. In the heavy-light configuration (figure $5 b^{\prime}$ ), the reflected shock RS1, weaker than its light-heavy analogue, would be noticeable by specifically tuning the contour levels. At later times (figures $\bar{x}$ and $\bar{x}^{\prime}$ ), the interface has evolved to a turbulent mixing zone that keeps growing while being processed by significantly weaker reshocks. In the heavy-light case (figure $5 c^{\prime}$ ), the main reshock TS1 travels slower in the heavy gas than its light-heavy analogue and has not exited the computational domain yet at that given time. The late-time light-heavy and heavy-light mixing layers resemble each other despite the differences already highlighted.

To distinguish the two configurations, we investigate the mean flow. The turbulent nature of the flow will be studied in Part 2. Figures 6 (log-linear), 7 and 8 (log-linear), respectively, depict the surface-averaged radial profiles of the primitive variables $\langle\rho\rangle(r, t), \widetilde{u}_{r}(r, t)$ and $\langle p\rangle(r, t)$, at different times. Density, radial velocity and pressure are made dimensionless using the light-fluid density $\rho_{L}=\min \left(\rho_{1_{0}}, \rho_{2_{0}}\right)$, the pressure ahead of the imploding shock $p_{0}$, and $\Delta u$. The pressure and radial velocity indicate the positions of the shocks, while the density profiles give additional information about the radial extent of the mixing layer. The first four time frames displayed characterize the imploding phase and show an increase in the shock strength. Post-shock values in the light-heavy case differ from the heavy-light ones since the converging shock travels in a fluid of different density and specific heat ratio. The fifth and sixth time frames represent the mean flow before and after the first reshock. Display of results at more intermediate times would confirm the existence of secondary reshocks. At late time, the mean flow is characterized by a radial velocity small compared with $\Delta u$, a quasi-constant background pressure and an asymmetric background density profile with a wide radial extent. The light-heavy and heavy-light configurations naturally present different final pressure levels and density stratifications.

Mean flow quantities have also been used to perform a preliminary grid convergence study. Three resolutions were considered for $\mathscr{V}_{1 / 8}: 128^{3}, 256^{3}$ (which has been used for deriving most of the statistics shown here) and $512^{3}$. Radial profiles of the primitive variables and the scalar field (scrutinized at a few given times) show that the $256^{3}$ and $512^{3}$ results are converged away from shock waves. As a result, we feel confident that the $256^{3}$ resolution for $\mathscr{V}_{1 / 8}$ (and consequently $512^{3}$ for $\mathscr{V}$ ) is satisfactory for the current investigation. This complements more exhaustive convergence studies already performed for simpler, canonical flows (e.g. Pantano et al. 2007). 

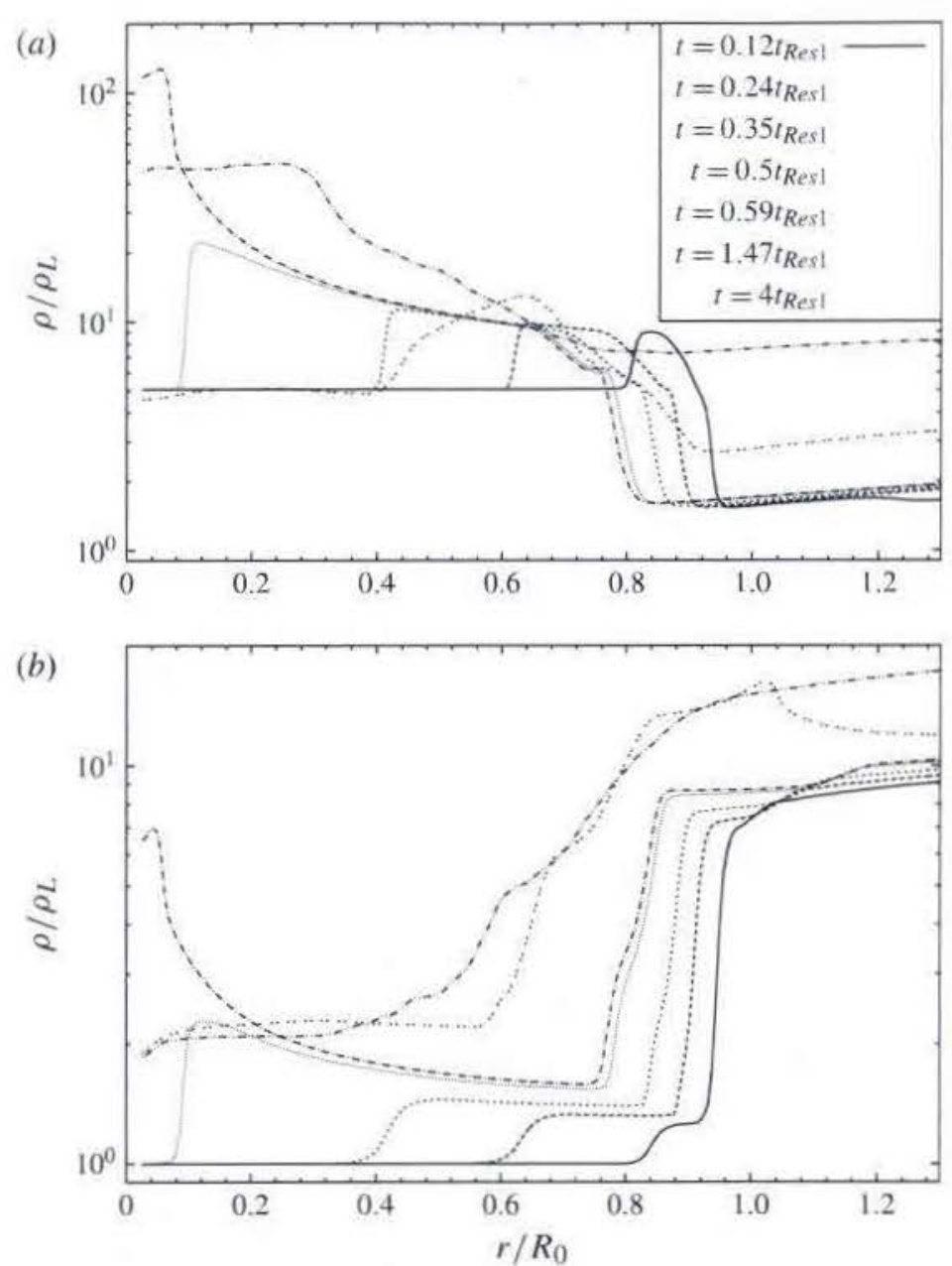

FIGURE 6. Surface-averaged radial profiles of density at different times: $(a)$ air $\rightarrow \mathrm{SF}_{6}$ and (b) $\mathrm{SF}_{6} \rightarrow$ air.

\section{Growth of the spherical mixing layer}

6.1. Spike, bubble, layer width and growth rate

The evolution of surface-averaged radial profiles of the scalar field $Y(r, t) \equiv$ $2 \psi(r, t)-1$ (figure 9) shows a developing asymmetry between the light- and heavy-fluid regions, particularly after the first reshock, as commonly observed in planar geometry when the Atwood ratio is large enough Lombardini et al. (2011). We define the spike, bubble and mixing-layer centre by the spherical surfaces of radii $r=r_{s}(t), r=r_{b}(t)$ and $r=r_{c}(t)$ such that $\langle\psi\rangle$ equals $1 \%, 99 \%$ and $50 \%$, respectively. The evolution of these radial locations and of the unperturbed position $R(t)$ are presented in figures 10 and 11 , indicating the various wave interactions (e.g. a first reshock time near $\Delta u t / R_{0} \approx 0.6$ in the light-heavy case and 0.27 in the heavy-light case), as well as an asymmetrical evolution of the spike and bubble structures. This asymmetry is also shown in the spike and bubble surface-averaged radial velocities (figure 12). This figure exhibits multiple impulsive accelerations of the density layer and different accelerating/decelerating phases, in particular periods when spikes are RT-stable while bubbles are RT-unstable, or vice versa, owing to the mixing-layer radial extent. 

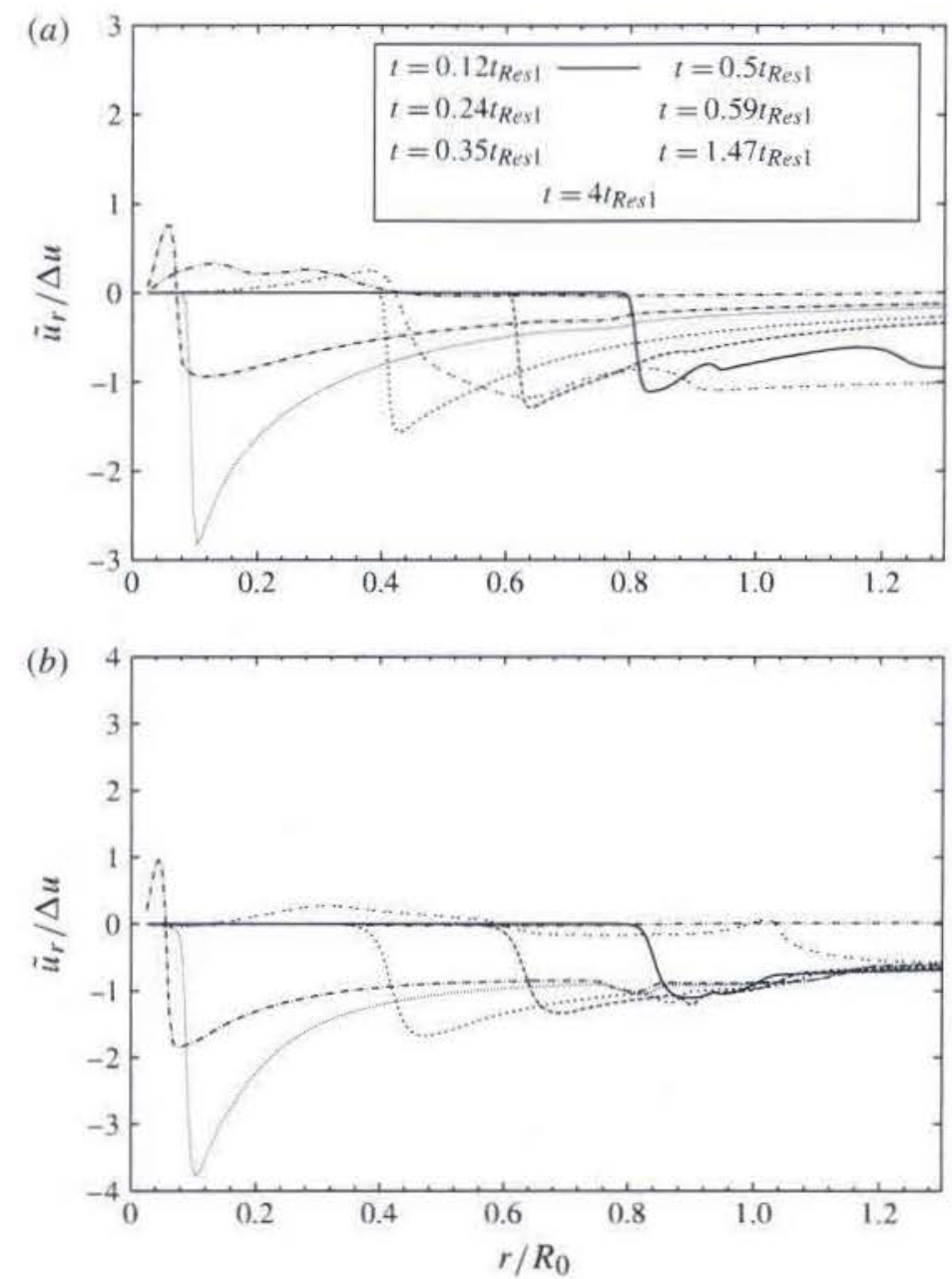

FIGURE 7. Surface-averaged radial profiles of radial velocity at different times: $(a)$ air $\rightarrow$ $\mathrm{SF}_{6}$ and $(b) \mathrm{SF}_{6} \rightarrow$ air.

To account for the mixing-layer growth, we prefer an integral definition of the mixing-layer width, rather than a threshold-based version that would consist of subtracting $r_{b}$ from $r_{s}$. This definition of the mixing-layer width is given by

$$
\delta(t)=\int_{0}^{\infty}\left(1-\langle Y\rangle^{2}\right) \mathrm{d} r .
$$

The width and growth rate evolution are displayed in figure 13. The first and second reshocks are well distinguished by sharp, negative values of the growth rate, e.g. at $\Delta u t / R_{0} \approx 0.6$ and 1.1 in the light-heavy case. In both configurations, the mixing-layer still grows significantly at late times. We can qualitatively compare the mixing-layer evolution with the LES data of Lombardini et al. (2011) obtained in planar geometry using similar initial perturbation shape and incident Mach number: at late times, the light-heavy planar growth slows down significantly more than what is observed in the spherical geometry, while the heavy-light planar growth resembles its spherical equivalent. The oscillating character of the growth of the light-heavy mixing-layer is due to two factors: (i) RT-unstable and stable phases that result from the oscillating 

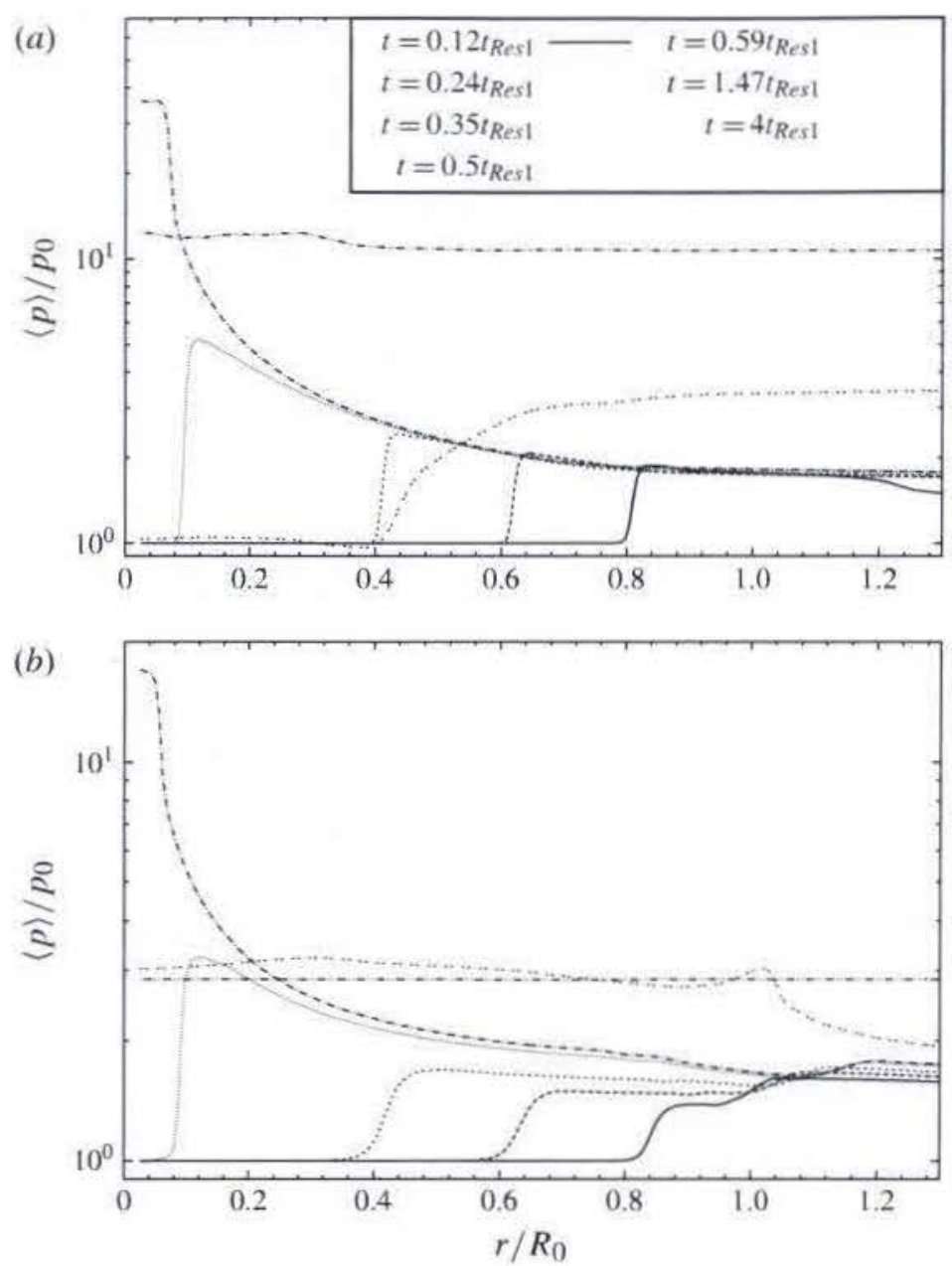

FIGURE 8. Surface-averaged radial profiles of pressure at different times: $(a)$ air $\rightarrow \mathrm{SF}_{6}$ and (b) $\mathrm{SF}_{6} \rightarrow$ air.

nature of the interface trajectory; (ii) phase reversal following each reshock, i.e. a decrease of the perturbation in amplitude before reversing its phase and growing further. We recall that the latter is typical of heavy-to-light shock interactions and can be explained on the basis of vorticity generation by baroclinic processes Meshkov (1969). Note that a phase reversal also appears when the converging shock initially impacts the heavy-light interface (figure $13 b$ ).

\subsection{Stability of perturbed spherical interfaces}

Baroclinic instabilities are nevertheless insufficient to fully understand the stability of fluid flows in radial motions. Consider for example an incompressible, thin spherical layer moving inward. By mass conservation, the layer thickens due to reduction of its circumference. This purely geometrical effect is known as the Bell-Plesset (BP) effect Bell (1951), Plesset (1954). The following theoretical development is inspired from $\mathrm{BP}$ analysis and used to draw qualitative conclusions on the growth of the spherical mixing layer. 

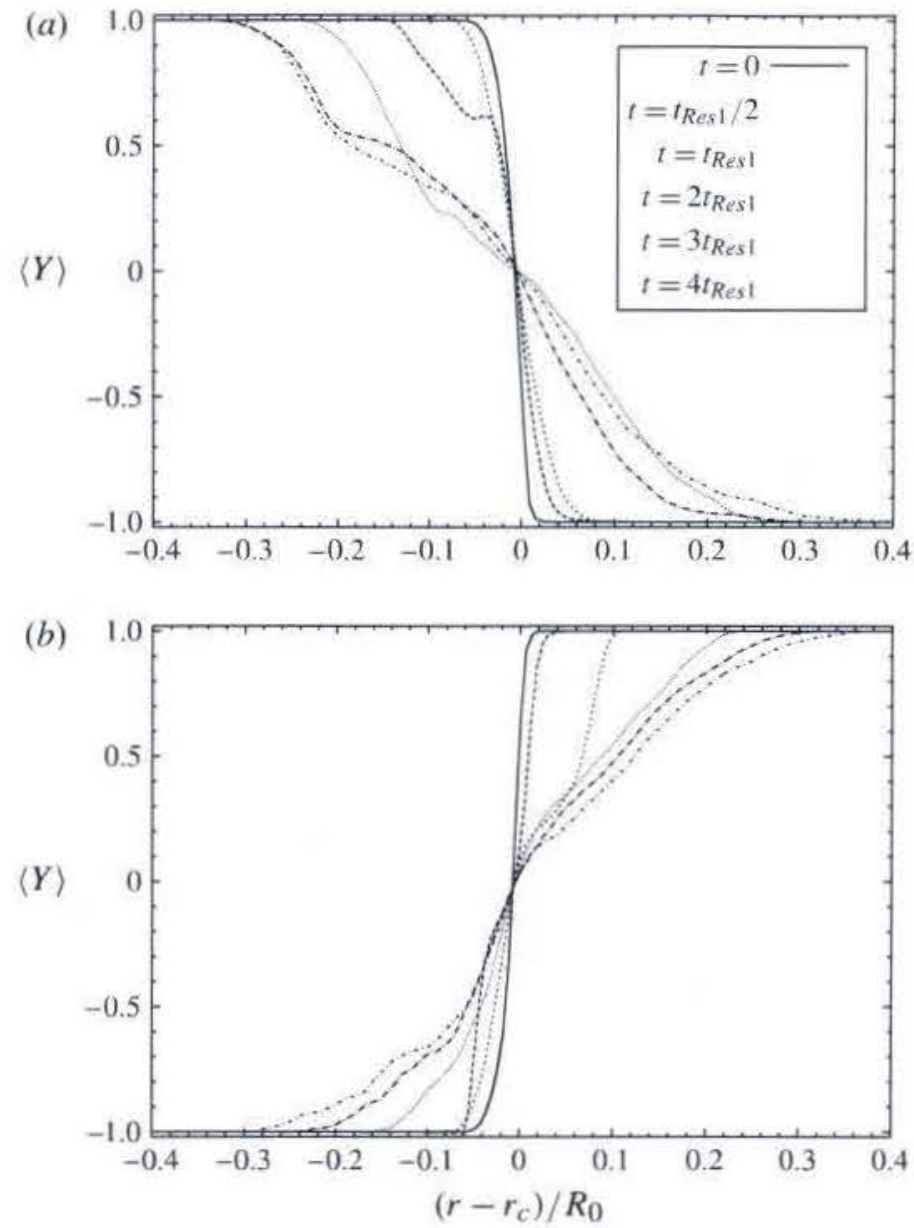

FIGURE 9. Surface-averaged scalar field $\langle Y\rangle$ radial profile (in the frame of the moving layer) at different times: $(a)$ air $\rightarrow \mathrm{SF}_{6}$ and $(b) \mathrm{SF}_{6} \rightarrow$ air.

\subsubsection{The base flow}

The problem of the linear stability of a harmonically perturbed contact discontinuity between two fluids of different densities in accelerated motion has been solved in a planar geometry by Taylor (1950). Postulating inviscid, incompressible, irrotational base flow and perturbations, Plesset (1954) extended the planar results to spherical symmetry, isolating the effect of the geometric convergence/divergence of the unperturbed interface on the perturbation growth, through the geometric convergence rate $\dot{R} / R$, where $R(t)$ represents the position of the unperturbed interface. Bell (1951) considered further the effect of compressibility by including a uniformly compressible base flow, but for free surfaces only, i.e. when $\left|A_{0}\right|=1$. The extension of Bell's treatment to interfaces with an arbitrary density jump is straightforward and the main results are summarized here. We recall Plesset's notation, consistent with our nomenclature, where the interface separates two fluids of uniform densities $\varrho_{1}(t)$ and $\varrho_{2}(t)$, ' 1 ' denoting the fluid contained within the sphere of radius $R$ and '2' denoting the fluid exterior to this sphere. The time-dependent Atwood ratio is then defined as $A=\left(\varrho_{1}-\varrho_{2}\right) /\left(\varrho_{1}+\varrho_{2}\right)$, and equals $A_{0}$ at $t<t_{0}$. The base velocity potential $\Phi_{i}(r, t)$ 

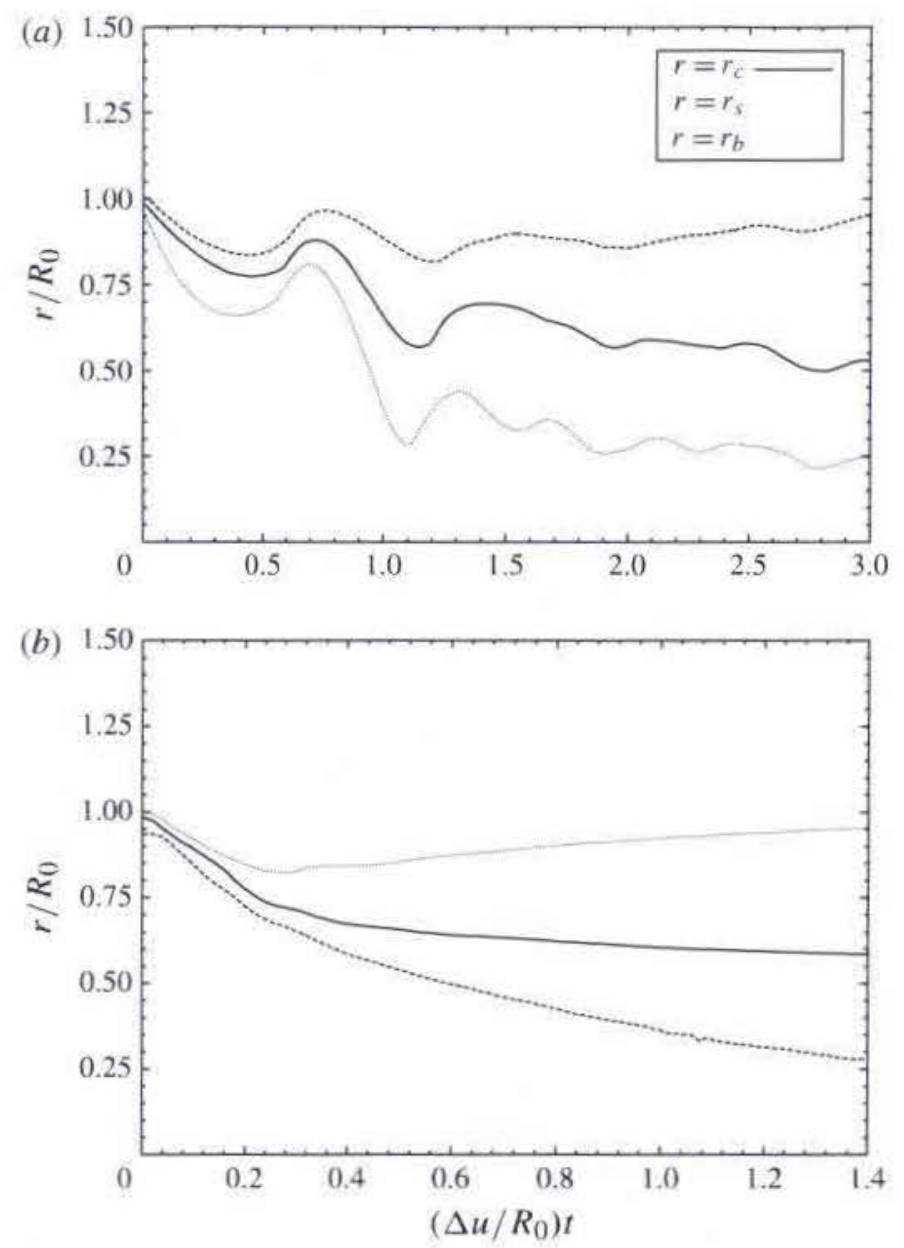

FIGURE 10. Spike (dashed curve), bubble (dotted curve) and mixing-layer centre (solid curve) radial positions versus $t:(a)$ air $\rightarrow \mathrm{SF}_{6}$ and $(b) \mathrm{SF}_{6} \rightarrow$ air.

satisfies, on each side of the interface,

$$
\nabla^{2} \Phi_{i}=\frac{\dot{\varrho}_{i}}{\varrho_{i}}-\frac{2}{3}\left(\frac{\dot{\varrho}_{1}}{\varrho_{1}}+3 \frac{\dot{R}}{R}\right) \frac{R^{3}}{r^{2}} \delta(r), \quad i=1,2,
$$

where the one-dimensional Dirac delta function $\delta(r)$ represents a mass source/sink at $r=0$ which makes it possible to specify uniform compression and geometric convergence rates independently. It is easy to verify that this radially symmetric base flow conserves mass everywhere. Solving (6.2) for $r>0$, one obtains on each side of the interface:

$$
\text { for all } r>0, \quad \Phi_{i}(r, t)=\frac{R^{2} \dot{R}}{r}+\frac{1}{3} \frac{\dot{\varrho}_{i}}{\varrho_{i}}\left(\frac{R^{3}}{r}+\frac{r^{2}}{2}\right), \quad i=1.2 .
$$

The first term represents Plesset's potential flow, the second term Bell's contribution. Bell's assumption of a uniformly compressible flow can be regarded as a reasonable approximation for the base flow in the vicinity of the perturbed interface. While the mass source/sink is a mathematically convenient representation of the centre of the 

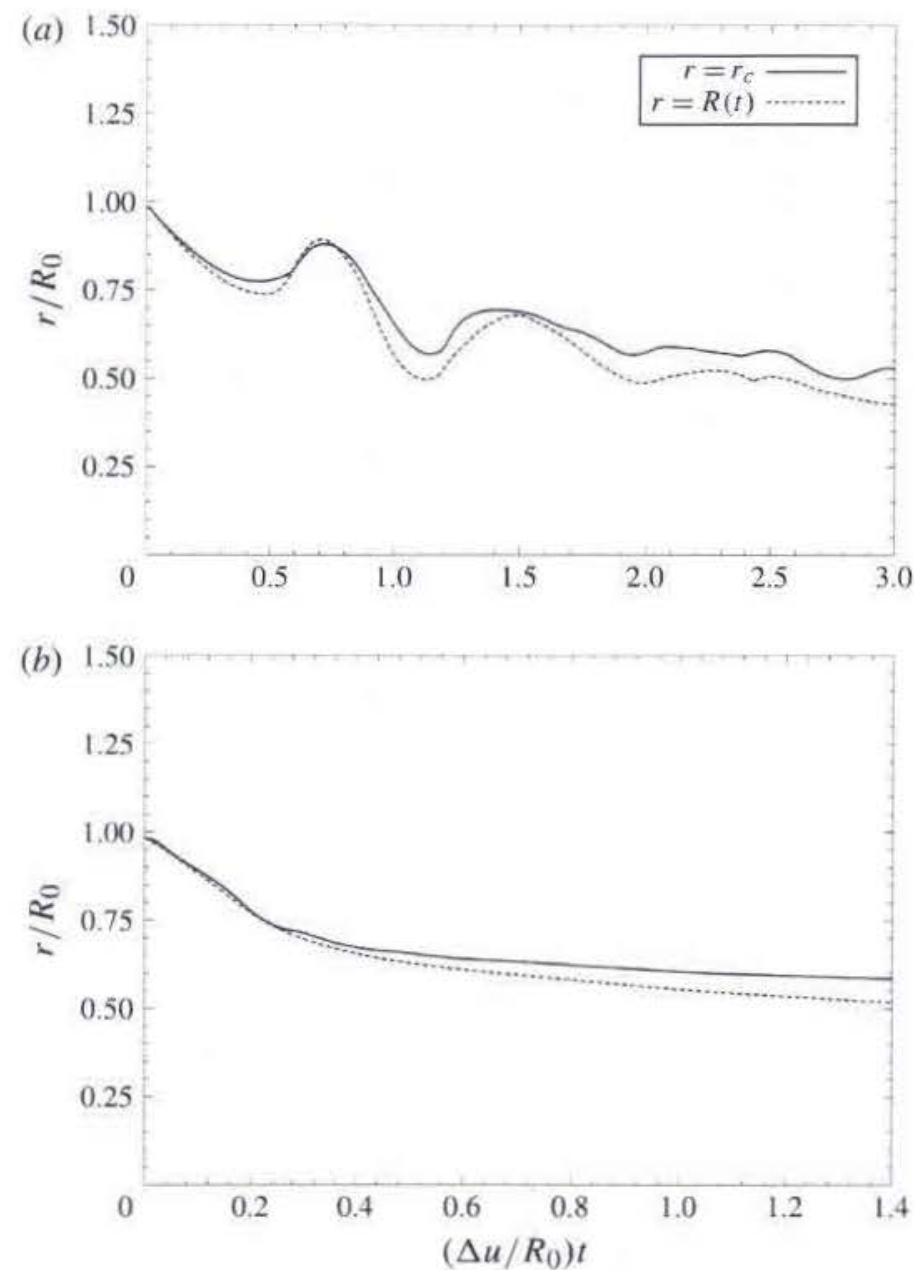

FIGURE 11. Mixing-layer centre (solid curve) and unperturbed interface (dashed curve) radial positions versus $t:(a)$ air $\rightarrow \mathrm{SF}_{6}$ and $(b) \mathrm{SF}_{6} \rightarrow$ air.

compressing fluid which allows for the decoupling between $\dot{\varrho} / \varrho$ and $\dot{R} / R$ near the interface, it would lead to unphysical pressures and erroneous results in the stability analysis when the interface is too close to the origin.

\subsubsection{Perturbation amplitude}

If the position of the perturbed interface is defined as

$$
\zeta(\theta, \phi ; t)=R(t)+a(t) Y_{\ell}^{m}(\theta, \phi),
$$

the amplitude $a(t)$ can be found by matching the base + perturbed pressure and normal velocity at $r=\zeta$, assuming incompressible perturbations. For small perturbations, linearization leads to the following ordinary differential equation:

$$
\begin{aligned}
\ddot{a} & +\left\{3 \frac{\dot{R}}{R}+\mathscr{R}_{\ell}\left[\frac{\dot{\varrho}_{1}}{\varrho_{1}}, \frac{\dot{\varrho}_{2}}{\varrho_{2}}\right]\right\} \dot{a}-\left\{\mathscr{R}_{\ell}[-\ell-2, \ell-1] \frac{\ddot{R}}{R}-\mathscr{R}_{\ell}\left[\frac{\dot{\varrho}_{1}}{\varrho_{1}}, \frac{\dot{\varrho}_{2}}{\varrho_{2}}\right] \frac{\dot{R}}{R}\right. \\
& \left.-\mathscr{R}_{\ell}\left[\frac{\mathrm{d}}{\mathrm{d} t}\left(\frac{\dot{\varrho}_{1}}{\varrho_{1}}\right), \frac{\mathrm{d}}{\mathrm{d} t}\left(\frac{\dot{\varrho}_{2}}{\varrho_{2}}\right)\right]\right\} a=0,
\end{aligned}
$$



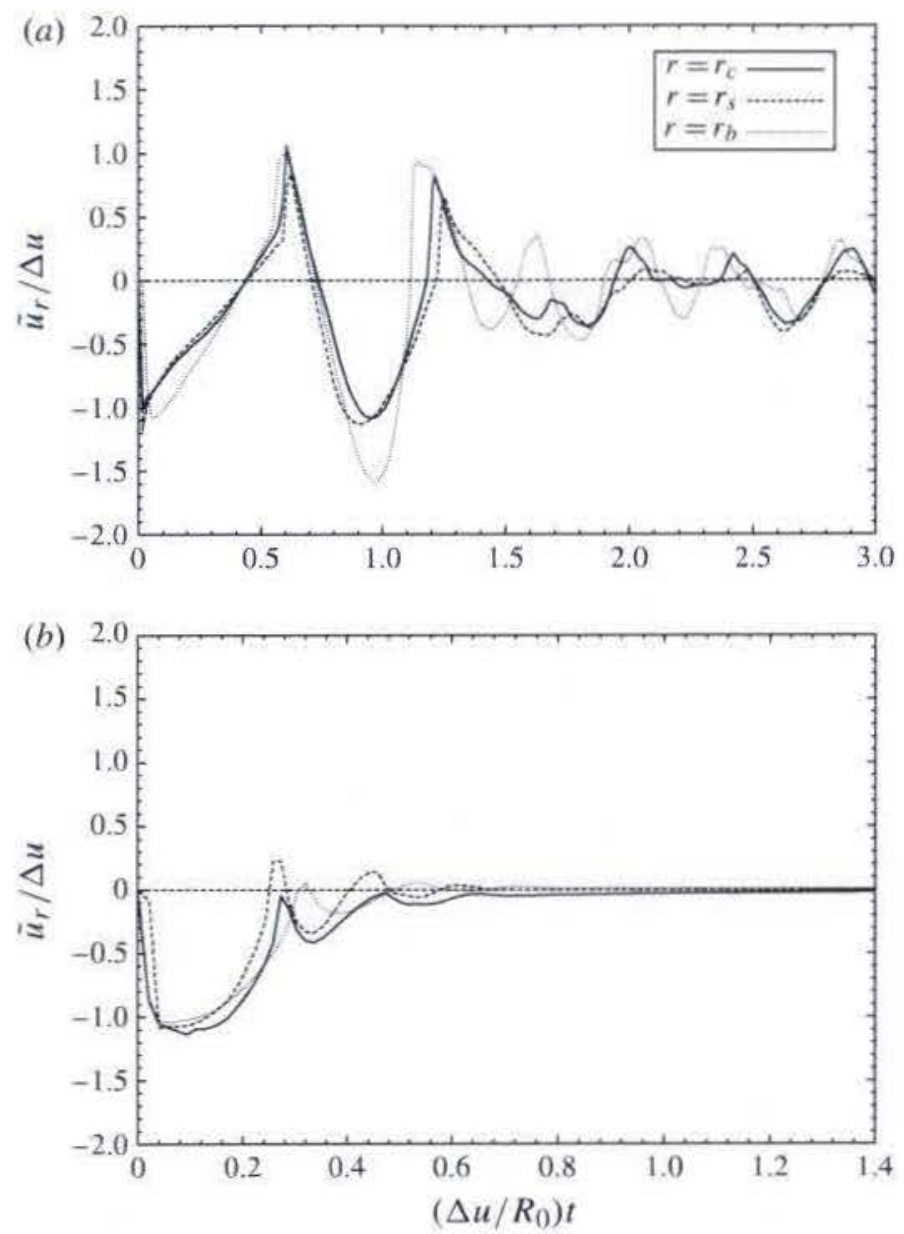

FIGURE 12. Spike (dashed curve), bubble (dotted curve) and mixing-layer centre (solid curve) surface-averaged radial velocities $\widetilde{u}_{r}$ versus $t:(a)$ air $\rightarrow \mathrm{SF}_{6}$ and (b) $\mathrm{SF}_{6} \rightarrow$ air.

where the two-variable function $\mathscr{R}_{\ell}$ is defined as the following weighted average:

$$
\mathscr{R}_{\ell}[\mathscr{X}, \mathscr{Y}]=\frac{(\ell+1) \varrho_{1} \mathscr{X}+\ell \varrho_{2} \mathscr{Y}}{(\ell+1) \varrho_{1}+\ell \varrho_{2}} \text {. }
$$

Making the following substitution

$$
a=\exp \left[-\frac{1}{2} \int_{0}^{t}\left\{3 \frac{\dot{R}}{R}+\mathscr{R}_{\ell}\left[\frac{\dot{\varrho}_{1}}{\varrho_{1}}, \frac{\dot{\varrho}_{2}}{\varrho_{2}}\right]\right\} \mathrm{d} t^{\prime}\right] \alpha=\sqrt{\frac{(\ell+1) \rho_{\mathrm{t}_{0}}+\ell \rho_{2_{0}}}{(\ell+1) \varrho_{1}+\ell \varrho_{2}}} \frac{R_{0}^{3}}{R^{3}} \alpha,
$$

equation (6.5) becomes

$$
\begin{aligned}
\ddot{\alpha}= & G(t) \alpha, \\
G(t)= & \left\{\mathscr{R}_{t}[-\ell-2, \ell-1]+\frac{3}{2}\right\} \frac{\ddot{R}}{R}+\frac{3}{4}\left(\frac{\dot{R}}{R}\right)^{2}+\frac{1}{2} \mathscr{R}_{\ell}\left[\frac{\ddot{\varrho}_{1}}{\varrho_{1}}, \frac{\ddot{\varrho}_{2}}{\varrho_{2}}\right] \\
& -\mathscr{R}_{\ell}\left[\frac{\mathrm{d}}{\mathrm{d} t}\left(\frac{\dot{\underline{\varrho}}_{1}}{\varrho_{1}}\right), \frac{\mathrm{d}}{\mathrm{d} t}\left(\frac{\dot{\varrho}_{2}}{\varrho_{2}}\right)\right]-\frac{1}{4} \mathscr{R}_{\ell}\left[\frac{\dot{\varrho}_{1}}{\varrho_{1}}, \frac{\dot{\varrho}_{2}}{\varrho_{2}}\right]^{2}+\frac{1}{2} \mathscr{R}_{\ell}\left[\frac{\dot{\varrho}_{1}}{\varrho_{1}}, \frac{\dot{\varrho}_{2}}{\varrho_{2}}\right] \frac{\dot{R}}{R} .
\end{aligned}
$$



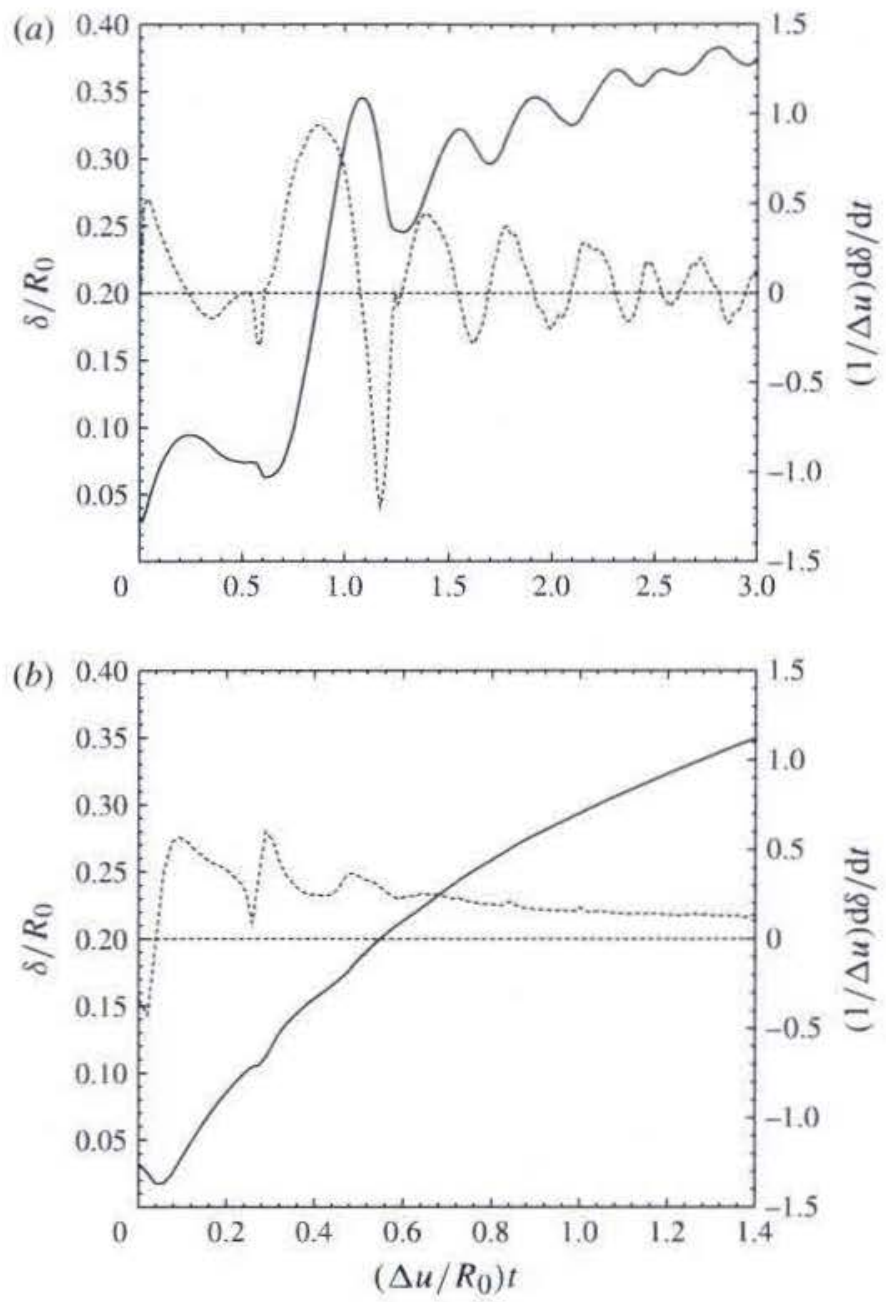

FIgURE 13. Mixing-layer width $\delta$ (solid curve) and growth rate $\mathrm{d} \delta / \mathrm{d} t$ (dashed curve) versus $t:(a)$ air $\rightarrow \mathrm{SF}_{6}$ and $(b) \mathrm{SF}_{6} \rightarrow$ air.

Assuming that the rate of compression applies everywhere, i.e. $\dot{\varrho}_{1} / \varrho_{1}=\dot{\varrho}_{2} / \varrho_{2} \equiv \dot{\varrho} / \varrho$, equation $(6.8 b)$ simplifies to

$$
G(t)=\underbrace{\left\{\mathscr{R}_{\ell}[-\ell-2, \ell-1]+\frac{3}{2}\right\} \frac{\ddot{R}}{R}}_{G_{\ddot{R}}(t)}+\underbrace{\frac{3}{4}\left(\frac{\dot{R}}{R}\right)^{2}}_{G_{R}(t)}+\underbrace{\frac{1}{4}\left(\frac{\dot{\varrho}}{\varrho}\right)^{2}-\frac{1}{2} \frac{\mathrm{d}}{\mathrm{d} t}\left(\frac{\dot{\varrho}}{\varrho}\right)}_{G_{\rho}(t)}+\underbrace{\frac{1}{2} \frac{\dot{\varrho}}{\varrho} \frac{\dot{R}}{R}}_{G_{\rho R}(t)} .
$$

From (6.7), the perturbation amplitude is then related to $\alpha(t)$ as

$$
a(t)=\beta(t) \alpha(t), \quad \text { with } \beta(t)=\sqrt{\frac{\varrho_{0} R_{0}^{3}}{\varrho R^{3}}},
$$

where the interface density $\varrho(t)$ can be taken as the arithmetic mean of $\varrho_{1}(t)$ and $\varrho_{2}(t)$, and $\varrho_{0} \equiv \varrho\left(t_{0}\right)$. It should be first noted that $\beta(t)$ is an (algebraic) stabilizing factor when $\varrho R^{3}$ is increasing with time, and destabilizing when $\varrho R^{3}$ is decreasing. Aside 
from this factor, the stability of the deformation (in the sense of exponential growth) is determined by the sign of $G(t)$ : the perturbation is unstable when $G(t)>0$.

\subsubsection{Limit cases}

In the expression (6.9), the component $G_{\ddot{R}}(t)$ represents the RT-like contribution to the growth rate, and reduces to RM growth if one chooses $\ddot{R}=\Delta u \delta(t)$, in which case $a(t)$ is actually linear in time, and not exponential. In the incompressible planar flow limit, which is reached when $\dot{\varrho}=\dot{R}=0$ and in the large wavenumber limit $\ell \gg 1, G_{\vec{R}}(t)$ is the only term contributing to $G(t)$ and naturally simplifies to $-(\ell / R) A \ddot{R}$, the square of the classical RT growth rate: the component $3 \ddot{R} /(2 R)$ which comes from taking the second derivative of $a$ in (6.7) is a purely geometric factor absent in planar geometry. RT stable and unstable scenarios will depend on the sign of $-A \ddot{R}$.

The second term, $G_{R}(t)$, which accounts for the geometric convergence, is always positive and contributes to the actual growth of the perturbation whether the base flow is converging or diverging. In the incompressible limit, $G_{\ddot{R}}(t)$ and $G_{R}(t)$ are the only terms contributing to $G(t)$ and Plesset's result is recovered. The sole term $G_{R}(t)$ can roughly characterize the zero-acceleration, quasi-incompressible 'coasting phase' of the ICF implosion, following the initial period of acceleration due to the driver and preceding the deceleration phase due to the compressing core.

When compression effects are considered, two additional terms, $G_{\rho}(t)$ and $G_{\rho R}(t)$, appear. In particular, Bell's result is recovered in the case of a free surface. The sign of the sum of both terms can be examined by rewriting the sum as

$$
G_{\rho}(t)+G_{\rho R}(t)=\frac{1}{4 \varrho R^{2}}\left(\frac{\dot{\varrho}}{\varrho}\right)^{3} \frac{\mathrm{d}}{\mathrm{d} t}\left[\varrho R^{2}\left(\frac{\varrho}{\grave{\varrho}}\right)^{2}\right] .
$$

Lastly, consider the zero-acceleration limit where no RT growth is allowed. Solving (6,5) directly leads to

$$
a(t)=a_{0} \frac{\varrho_{0} R_{0}^{2}}{\varrho R^{2}}\left[1+\left.\frac{\dot{z}}{z}\right|_{t=t_{0}} \int_{0}^{t} \frac{\varrho R}{\varrho_{0} R_{0}} \mathrm{~d} t^{\prime}\right], \quad \text { with } \frac{\dot{z}}{z}=\frac{\dot{\varrho}}{\varrho}+2 \frac{\dot{R}}{R}+\frac{\dot{a}}{a} .
$$

\subsubsection{Application to the spherical layer growth}

One can determine the factors $\beta(t)$ and $G(t)$, and solve for $a(t)$, by specifying independently the base flow histories $(\dot{\varrho} / \varrho)(t)$ and $R(t)$, and given $\ell$ and the initial values $\rho_{\mathrm{I}_{0}}, \rho_{2_{0}}, R_{0}$ and $a_{0}$. To study the BP effects on the mixing-layer growth, we will actually identify the base flow with the surface-averaged quantities obtained from the LES, and $R(t)$ to the mixing-layer centre $r_{c}(t)$. Alternately, $\beta$ and $G$ could be evaluated from a radially symmetric flow simulation, which would lead to comparable results. In fact, neither the surface-averaged LES nor the radially symmetric flow are well approximated by a uniformly compressible base flow everywhere in radius. The effects of the density radial stratification are indeed ignored in the stability analysis, but this simplified reasoning can still serve as a guide for comparing BP effects with RT/RM instabilities within the mixing layer. Another limitation of applying the results of the stability analysis to the rate of development of interface distortions of significant amplitude lies in the initial assumption of small perturbations.

The term $G_{\vec{R}}$ in (6.9) was evaluated by identifying $\varrho_{1}(t)$ and $\varrho_{2}(t)$ to $\langle\rho\rangle(r, t)$ at $r=r_{b}(t)$ and $r_{c}(t)$, or $r=r_{c}(t)$ and $r_{b}(t)$, depending on the sign of $A$. To avoid differentiating the interface radial velocity with respect to time in the evaluation of 

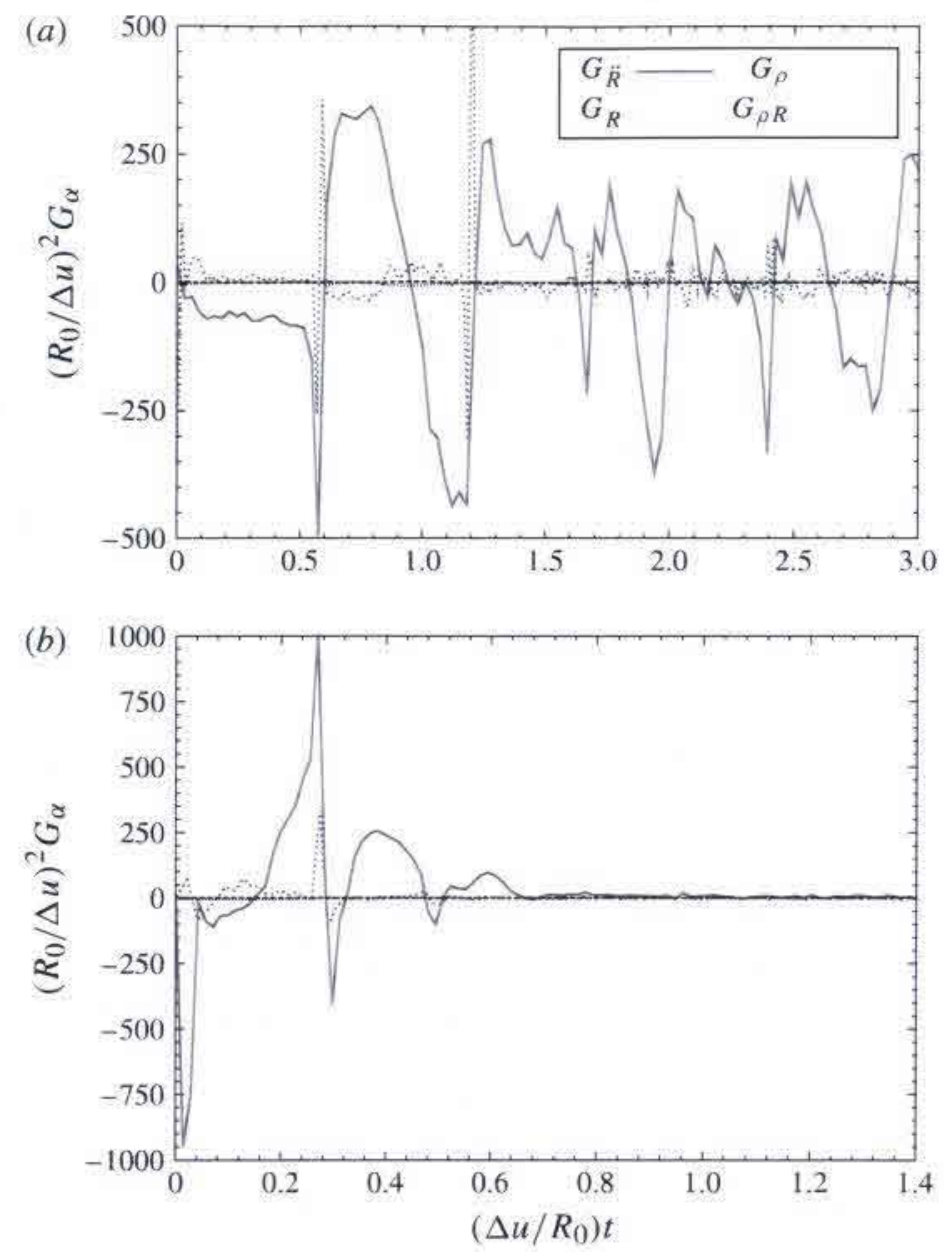

FIGURE 14. (Colour online) Growth function components $G_{\ddot{R}}, G_{R}, G_{\rho}$ and $G_{\rho R}$ versus $t$ : (a) air $\rightarrow \mathrm{SF}_{6}$ and $(b) \mathrm{SF}_{6} \rightarrow$ air. See $\$ 6.2$ for definitions.

$\ddot{R}$, we observe that the radial symmetric flow satisfies $\ddot{R}=-(1 / \rho) \partial P / \partial r$, and $\ddot{R}$ was therefore determined as $\langle-(1 / \rho) \partial P / \partial r\rangle\left(r_{c}, t\right)$. For similar reasons, the compression ratio $\dot{\varrho} / \varrho$ in the terms $G_{\rho}$ and $G_{\rho R}$ was computed as $-\langle\nabla \cdot \boldsymbol{u}\rangle\left(r_{c}, t\right)$.

The perturbation growth is given by the exponential growth factor $G(t)$ defined by $(6.9)$ and the algebraic amplification factor $\beta(t)$ defined by (6.10). Figure 14 represents the evolution of each term in (6.9). Positive values would contribute to the layer growth. The first observation is that the RT-like contribution $G_{\vec{R}}$ is the most dominant term, because of the initial choice of large wavenumbers $\ell_{0}=40$. By large $\ell_{0}$ it is meant here that the characteristic initial perturbation wavelength is small compared with $R_{0}$. The local perturbation wavelength does not see the effect of the curvature of the converging flow, aside from the acceleration of the layer. One can actually estimate the magnitude of $G_{R}, G_{\rho}$ and $G_{\rho R}$ with respect to $G_{\ddot{R}}$ in the limit $\ell \gg 1$ : assigning the same time scale for the time derivatives, $G_{R} \sim G_{\rho} \sim G_{\rho R} \sim G_{\ddot{R}} /(\ell A)$. In addition, we confirm the intuition developed earlier that the RT effect on the growth remains significant at late times in the light-heavy configuration. Finally, we observe that the second largest contribution is due to the purely compressible nature of the radial flow $\left(G_{\rho}\right)$. There exist RT-stable regions where this term contributes positively to $G$. Plesset's purely geometric contribution $\left(G_{R}\right)$ is negligible compared to Taylor's 

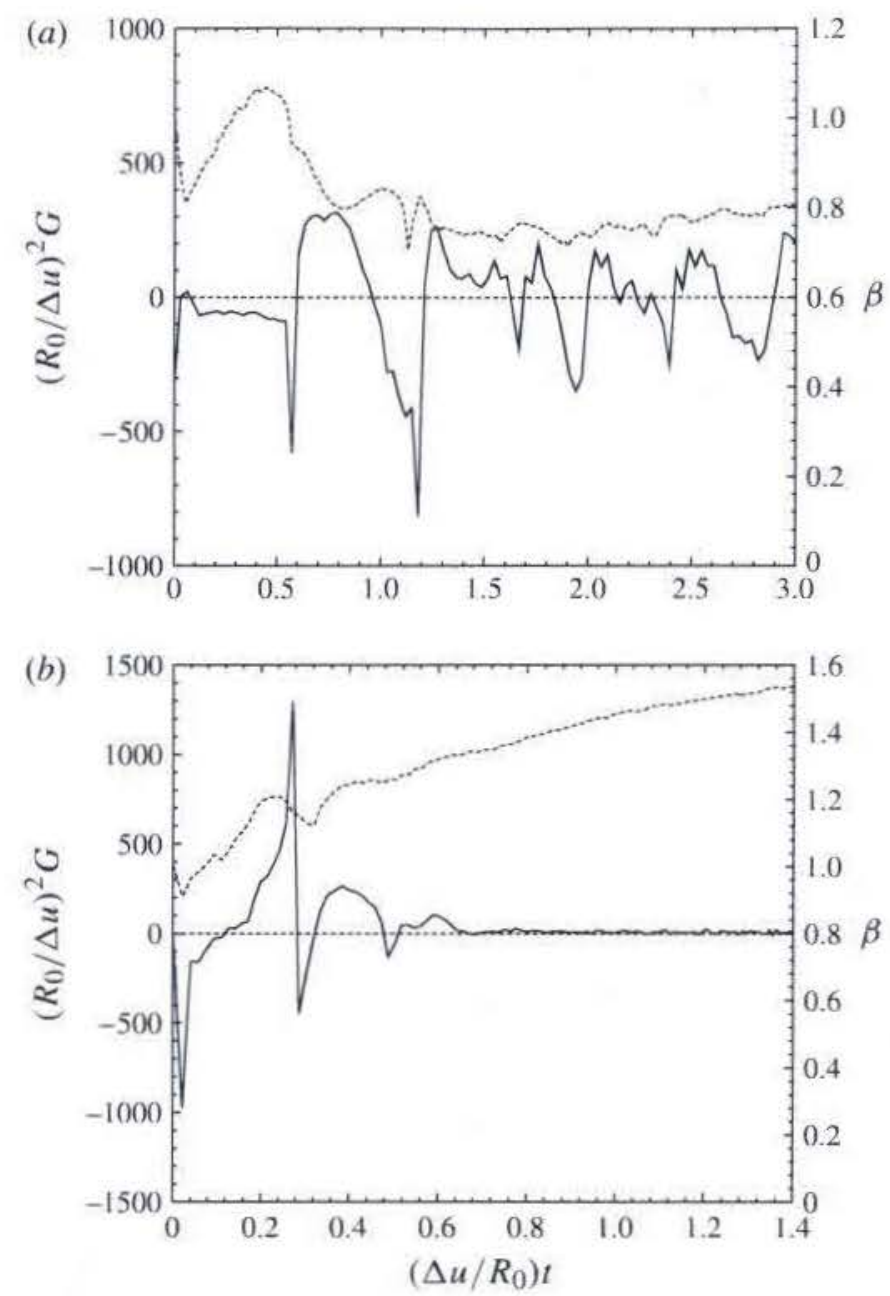

FIGURE 15. Growth function $G$ (solid curve) and factor $\beta$ (dashed curve) versus $t$ : (a) air $\rightarrow \mathrm{SF}_{6}$ and $(b) \mathrm{SF}_{6} \rightarrow$ air. See $\S 6.2$ for definitions.

instability and Bell's compressible effects. The sum $G$ of these terms is shown in figure 15, together with the evolution of $\beta$, and confirms the dominance of the RT contribution. The effect of $\beta$ is essentially destabilizing prior to the first reshock in the light-heavy case, whereas its effect is destabilizing at almost all times in the heavy-light configuration.

\section{Summary of results}

We have performed a series of exploratory, low-numerical dissipation LES using an explicit structural SGS model that computes the mixing generated at a spherical density interface impacted by a converging shock. The shock has been generated using a self-similar solution of the Euler equations, and a statistically isotropic, initial perturbation with a dominant wavelength $\ll R_{0}$ has been tailored using spherical harmonics. While the shock Mach number and perturbation shape have been kept fixed, we have explored two canonical configurations: the converging shock travelling from the light to the heavy fluid (i.e. light-heavy case), or vice versa (i.e. heavy-light case). We investigated the effect of the geometry on the layer growth by looking at 
$(r, t)$ wave diagrams as well as performing a stability analysis of a linearly perturbed spherical interface, following closely the work of Bell (1951) and Plesset (1954). The analysis essentially results in a growth factor that nicely decouples the following important effects: RM/RT instability, geometric convergence and compressibility. Each term can be quantified from the mean (surface-averaged) flow. The other novelty here is the inclusion of geometric and compressible effects for density interfaces of $A_{0} \neq 0$.

The following conclusions are relevant to incident implosions with shocks of moderate Mach number at impact and for the presently defined class of interface perturbations.

(i) The heavy-light flow exhibits the same wave refractions observed in its planar analogue: the imploding shock, after reflection off the origin, reshocks the interface in a light-to-heavy fashion, producing a reflected shock which, in turn, reflects off the origin and impacts the interface during a second reshock event. Successive reshocks of decreasing intensity follow. In the light-heavy case, a rarefaction wave is reflected inwards following the first reshock interaction. However, unlike in planar geometry, a shock forms at the tail of the rarefaction wave, due to an over-expansion of the flow. This secondary shock is responsible for a second reshock event. A reflected rarefaction wave is reflected and a shock forms at its tail, which then leads to a third reshock, etc.

(ii) In the event that the interface is perturbed, each shock interaction is expected to cause RM growth. The $(r, t)$ wave diagrams also indicate the phases where the interface accelerates and decelerates, hence RT-unstable regions. There exists no equivalent RT mixing in planar shock-driven mixing. In the light-heavy (heavy-light) case, the interface is RT unstable if it accelerates inward (outward) or decelerates outward (inward). The growth rate, measured from the surface-averaged mass fraction field, confirmed the differences observed between the light-heavy and heavy-light radially symmetric flow.

(iii) Lombardini (2008) observed that, in the cylindrical geometry, the wave diagrams for the light-heavy and heavy-light configurations show the same characteristics as the spherical geometry (similar reshock formations and RT-unstable regions).

(iv) Considering the rather small initial perturbation wavelengths considered (compared with the initial radius) as $\ell_{0}=40$, baroclinic instabilities are the dominant effect on the mixing-layer growth. Similarly, the ICF implosion simulations of Thomas \& Kares (2012) have shown that RM/RT instabilities are largely responsible for the amplification of asymmetries of comparable mode number $(\ell=30)$ even at higher convergence ratios $(\approx 8)$. As suggested in the ICF experiments by $\mathrm{Li}$ et al. (2004), BP effects are expected to become important at much higher convergence ratios $(30-40)$ and low-mode-number asymmetries $(\ell<10)$ such as those resulting from capsule fabrication asymmetry or drive-pressure asymmetry (due to non-uniformity in on-target laser intensity).

(v) Surprisingly, the second most important effect is presently found to be the compressible nature of the mean flow, and not the geometric convergence. For the incident Mach number used presently, the mixing-layer centre reached a convergence ratio $R_{0} / r_{c}(t \rightarrow \infty) \approx 2$ in both light-heavy and heavy-light cases. At higher incident Mach numbers, the initial interface follows the transmitted shock TS0 more closely than at lower Mach numbers and is therefore reshocked closer to the centre, leading to a higher final convergence ratio Lombardini (2008). 
(vi) The analysis assumes initial perturbations of small amplitude compared with the characteristic perturbation wavelength. Although we start with an initial amplitude-to-wavelength ratio of approximately $20 \%$ in the simulation, other ratios should be studied. The relatively large amplitude of the perturbation was actually chosen to more clearly display the turbulent mixing arising from the imposed asymmetry at the small convergence ratio achieved in these simulations. More realistic convergence ratios would require a few additional levels of mesh refinement or a more refined base grid.

Part 2 investigates the turbulent nature of the mixing, with a focus on the spectral analysis of turbulent statistics on spherical surfaces taken within the mixing layer (Lombardini et al. 2014).

\section{Acknowledgement}

This work has been supported in part by the Department of Energy under subcontract no. DE-AC52-06NA25396.

\section{REFERENCES}

BALAKRishnan, K. \& MENON, S. 2011 Characterization of the mixing layer resulting from the detonation of heterogeneous explosive charges. Flow Turbul. Combust. 87, 639-671.

Barnes, C. W., Batha, S. H., Dunne, A. M., Magelssen, G. R., Rothman, S., Day, R. D., Elliott, N. E., Haynes, D. A., Holmes, R. L., Scott, J. M., Tubbs, D. L., Youngs, D. L., Boehly, T. R. \& JAanimagi, P. 2002 Observation of mix in a compressible plasma in a convergent cylindrical geometry. Phys. Plasmas 9 (11), 4431-4434.

BELL, G. I. 1951 Taylor instability on cylinders and spheres in small amplitude approximation. Los Alamos Scientific Laboratory Report, LA-1321.

Berger, M. J. \& Colella, P. 1989 Local adaptive mesh refinement for shock hydrodynamics. J. Comput. Phys. 82 (1), 64-84.

ChISNELL, R. F. 1998 An analytic description of converging shock waves. J. Fluid Mech. 354, $357-375$.

COOK, A. W., САвот, W. \& Miller, P. L. 2004 The mixing transition in Rayleigh-Taylor instability. J. Fluid Mech. 511, 333-362.

DEITERDING, R. 2005 Construction and application of an AMR algorithm for distributed memory computers. In Adaptive Mesh Refinement - Theory and Applications (ed. T. Plewa, T. Linde \& V. G. Weirs), Lecture Notes in Computational Science and Engineering, vol. 41, pp. 361-372. Springer.

Fedkiw, R. P., Aslam, T, Merriman, B. \& OSher, S. 1999 A non-oscillatory Eulerian approach to interfaces in multimaterial flows (the ghost fluid method). J. Comput. Phys. 152 (2), 457-492.

FRIEDMAN, M. P. 1960 An improved perturbation theory for shock waves propagating through non-uniform regions. J. Fluid Mech. 8, 193-209.

Gittings, M., Weaver, R., Clover, M., Betlach, T., Byrne, N., Coker, R., Dendy, E., Hueckstaedt, R., New, K., OAKes, W. R., Ranta, D. \& Stefan, R. 2008 The Rage radiation-hydrodynamic code. Comput. Sci. Disc. 1 (1), 015005.

Glimm, J., Grove, J. W., Zhang, Y. \& Dutta, S. 2002 Numerical study of axisymmetric Richtmyer-Meshkov instability and azimuthal effect on spherical mixing. J. Stat. Phys. 107 $(1 / 2), 241-260$.

GotTlieb, S., Shu, C. -W. \& TADMOR, E. 2001 Strong stability-preserving high-order time discretization methods. SIAM Rev. 43 (1), 89-112.

GUderLey, G. 1942 Starke Kugelige und zylindrische Verdichtungsstösse in der Nähe des Kugelmittelpunktes bzw der Zylinderachse. Luftfahrtforschung 19, 302-312. 
HiLl, D. J. \& PUllin, D. I. 2004 Hybrid tuned centre-difference-WENO method for large eddy simulations in the presence of strong shocks. J. Comput. Phys. 194 (2), 435-450.

Honein, A. E. \& MoIN, P. 2004 Higher entropy conservation and numerical stability of compressible turbulence simulations. J. Comput. Phys. 201 (2), 531-545.

Hosseini, S. H. R. \& TAKAYAMA, K. 2005 Experimental study of Richtmyer-Meshkov instability induced by cylindrical shock waves. Phys. Fluids 17 (8), 084101.

Joggerst, C. C., Almgren, A.\& Woosley, S. E. 2010 Three-dimensional simulations of RayleighTaylor mixing in core-collapse supernovae. Astrophys. J. 723, 353-363.

JUN, B., JONES, T. W. \& NORMA, M. L. 1996 Interaction of Rayleigh-Taylor fingers and circumstellar cloudlets in young supernova remnants. Astrophys. J. 468, 59-63.

KRECHETNIKOV, R. 2009 Rayleigh-Taylor and Richtmyer-Meshkov instabilities of flat and curved interfaces. J. Fluid Mech. 625, 387-410.

KRECHETNIKOV, R. \& HOMSY, G. M. 2009 Crown-forming instability phenomena in the drop splash problem. J. Colloid Interface Sci. 331, 555-559.

Kumar, S., Hornung, H. G. \& Sturtevant, B. 2003 Growth of shocked gaseous interfaces in a conical geometry. Phys. Fluids 15 (10), 3194-3208.

Li, C. K., Séguin, F. H., Frenje, J. A., Petrasso, R. D., Delettrez, J. A., Mckenty, P. W., Sangster, T. C., Keck, R. L., Soures, J. M., Marshall, F. J., Meyerhofer, D. D., Goncharov, V. N., Knauer, J. P., Radha, P. B., Regan, S. P. \& Seka, W. 2004 Effects of nonuniform illumination on implosion asymmetry in direct-drive inertial confinement fusion. Phys. Rev. Lett. 92, 205001.

Lin, H., Storey, B. D. \& SZERI, A. J. 2002 Rayleigh-Taylor instability of violently collapsing bubbles. Phys. Fluids 14 (8), 2925-2928.

LINDL, J. D. 1998 Inertial Confinement Fusion: The Quest for Ignition and Energy Gain using Indirect Drive. Springer.

LOMBARDINI, M. 2008 Richtmyer-Meshkov instability in converging geometries. PhD Thesis, California Institute of Technology. http:/thesis.library.caltech.edu/2319\%.

Lombardini, M. \& DeITERDing, R. 2010 Large-eddy simulations of Richtmyer-Meshkov instability in a converging geometry. Phys. Fluids 22 (9), 091112.

Lombardini, M., Hill, D. J., Pullin, D. I. \& Meiron, D. I. 2011 Atwood ratio dependence of Richtmyer-Meshkov flows under reshock conditions using large-eddy simulations. J. Fluid Mech. 670, 439-480.

Lombardini, M. \& PUllin, D. I. 2009 Small-amplitude perturbations in the three-dimensional cylindrical Richtmyer-Meshkov instability. Phys. Fluids 21 (11), 114103.

Lombardini, M., PUllin, D. I. \& MEIRon, D. I. 2012 Transition to turbulence in shock-driven mixing: a Mach number study. J. Fluid Mech. 690, 203-226.

Lombardini, M., Pullin, D. I. \& Meiron, D. I. 2014 Turbulent mixing driven by spherical implosions. Part 2. Turbulence statistics. J. Fluid Mech. 748, 113-142.

MANKBADi, M. R. \& BALACHANDAR, S. 2012 Compressible inviscid instability of rapidly expanding spherical material interfaces. Phys. Fluids 24 (3), 034106.

Meshrov, E. E. 1969 Instability of the interface of two gases accelerated by a shock wave. Sov. Fluid Dyn. 4 (5), 101-108.

Mikaelian, K. O. 1990 Rayleigh-Taylor and Richtmyer-Meshkov instabilities and mixing in stratified spherical shells. Phys. Rev. A 42, 3400-3420.

MIKAELIAN, K. O. 2005 Rayleigh-Taylor and Richtmyer-Meshkov instabilities and mixing in stratified cylindrical shells. Phys. Fluids 17 (9), 094105.

Misra, A. \& Pullin, D. I. 1997 A vortex-based model for large-eddy simulation. Phys. Fluids 9 (8), 2443-2454.

Motl, B., Oakley, J., Ranjan, D., Weber, C., Anderson, M. \& Bonazza, R. 2009 Experimental validation of a Richtmyer-Meshkov scaling law over large density ratio and shock strength ranges. Phys. Fluids 21 (12), 126102.

Orlicz, G. C., Balakumar, B. J., Tomkins, C. D. \& Prestridge, K. P. 2009 A Mach number study of the Richtmyer-Meshkov instability in a varicose, heavy-gas curtain. Phys. Fluids 21 (6), 064102 . 
Pantano, C., Deiterding, R., Hill, D. J. \& Pullin, D. I. 2007 A low numerical dissipation patch-based adaptive mesh refinement method for large-eddy simulation of compressible flows. J. Comput. Phys. 221 (1), 63-87.

Plesset, M. S. 1954 On the stability of fluid flows with spherical symmetry. J. Appl. Phys, 25, 96-98.

Pullin, D. I. 2000 A vortex-based model for the subgrid flux of a passive scalar. Phys. Fluids 12 (9), 2311-2319.

RICHTMYER, R. D. 1960 Taylor instability in shock acceleration of compressible fluids. Commun. Pure Appl. Maths 13, 297-319.

TAYLOR, G. I. 1950 The instability of liquid surfaces when accelerated in a direction perpendicular to their planes. Proc: R. Soc: Lond. A 201, 192-196.

THOMAS, V. A. \& KARES, R. J. 2012 Drive asymmetry and the origin of turbulence in an ICF implosion. Phys. Rev. Lett. 109 (7), 075004.

Thornber, D., Drikakis, D. L., Youngs, D. L. \& Williams, R. J. R. 2010 The influence of initial conditions on turbulent mixing due to Richtmyer-Meshkov instability. J. Fluid Mech. 654, 99-139.

Vandenboomgaerde, M., Mügler, C. \& Gauthier, S. 1998 Impulsive model for the RichtmyerMeshkov instability. Phys. Rev. E 58 (2), 1874-1882.

Vetter, M. \& Sturtevant, B. 1995 Experiments on the Richtmyer-Meshkov instability of an air $/ S F_{6}$ interface. Shock Waves 4, 247-252.

WeCKen, F. 1950 Expansion einer Gaskugel hohen Druckes. Z. Angew. Math. Mech. 30, $270-271$.

Welser-Sherrill, L., Haynes, D. A., Mancini, R. C., Cooley, J. H., Tommasini, R., Golovkin, I. E., Sherrill, M. E. \& HaAn, S. W. 2008 Inference of ICF implosion core mix using experimental data and theoretical mix modelling. High Energ. Dens. Phys. 5 (4), 249-257.

Youngs, D. L. \& Williams, R. J. R. 2008 Turbulent mixing in spherical implosions. Intl J. Numer. Meth. Fluids 56 (8), 1597-1603.

YU, H. \& LIVESCU, D. 2008 Rayleigh-Taylor instability in cylindrical geometry with compressible fluids. Phys. Fluids 20 (10), 104103.

ZHANG, Q. \& GRAHAM, M. J. 1998 A numerical study of Richtmyer-Meshkov instability driven by cylindrical shocks. Phys. Fluids 10 (4), 974-992. 